NASA Technical Memorandum 104413

$1 N-34$

ICOMP-91-09; CMOTT-91-03

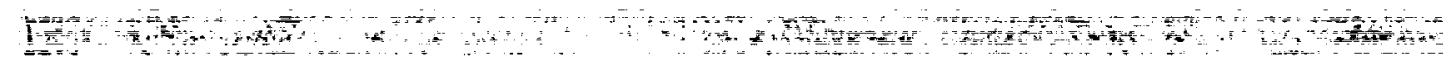 \\ Advances in Modeling the Pressure Correlation Terms in the Second Moment Equations
}

Tsan-Hsing Shih and Aamir Shabbir

Institute for Computational Mechanics in Propulsion

and Center for Modeling of Turbulence and Transition

Lewis Research Center

Cleveland, Ohio

and

John L. Lumley

Cornell University

Ithaca, New York

June 1991 


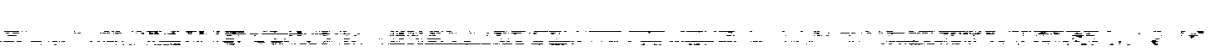




\title{
ADVANCES IN MODELING THE PRESSURE CORRELATION TERMS IN THE SECOND MOMENT EQUATIONS
}

\author{
Tsan-Hsing Shih and Aamir Shabbir \\ Institute for Computational Mechanics in Propulsion \\ and Center for Modeling of Turbulence and Transition \\ Lewis Research Center \\ Cleveland, Ohio 44135 \\ and \\ John L. Lumley \\ Comell University \\ Ithaca, New York 14853
}

\begin{abstract}
In developing turbulence models, different authors have proposed various model constraints in an attempt to make the model equations more general (or universal). The most recent of these are the realizability principle (Lumley 1978, Schumann 1977), the linearity principle (Pope 1983), the rapid distortion theory (Reynolds 1987) and the material indifference principle (Speziale 1983). In this paper we will discuss sevcral issues concerning these principles and will pay special attention to the realizability principle raised by Lumley (1978). Realizability (defined as the requirement of non-negative energy and Schwarz' inequality between any fluctuating quantities) is the basic physical and mathematical principle that any modeled equation should obey. Hence, it is the most universal, important and also the minimal requirement for a model equation to prevent it from producing unphysical results. In this paper we will describe in detail the principle of rcalizability, derive the realizability conditions for various turbulence models, and propose the model forms for the pressure correlation terms in the second moment equations. Detailed comparisons of various turbulence models (Launder et al. 1975, Craft et al. 1989, Zeman and Lumley 1976, Shih and Lumley 1985 and one proposed here) with experiments and direct numerical simulations will be presented. As a special case of turbulence, we will also discuss the two-dimensional two-component turbulence modeling.
\end{abstract}




\section{Introduction}

It is commonly accepted that the Navier-Stokes is the basic equation governing turbulence. Hence, without any model approximations, direct numerical simulation (DNS) should be an ideal approach to study all the aspects of turbulence. For example, using DNS we may study the dynamics of various turbulence structures; the mechanisms of energy transfer; and the interaction between different size of eddies; and we may also look at the various one-point and two-point statistics. Unfortunately, DNS is limited to relatively low Reynolds number and simpler flows due to a wide range of length and time scales that are present in a turbulent flow and due to today's computational limitations (both computer hardware and numerical methods). Therefore, at present, DNS is far from computing the practical engineering problems. For solving general engineering problems, turbulence modeling must be used in conjunction with the CFD. The history and the literature of turbulence modeling is described in detail by Lumley $(1978,1983)$, Reynolds $(1976)$, Launder (1989) and Rodi (1988). All the model equations were designed to approximate the Navier-Stokes equations. The model coefficients (or constants) have been tuned against some basic turbulent flows. The accuracy of any model equation (if it works) varies from flow to flow. In addition, we must use them with caution because any model equation will miss some of the physics or violate some of the properties of the Navier-Stokes equations because of the approximations made. Results in such cases may be misleading in studying unknown turbulence phenomena. More severely, some of the modcl cquations may end up with unphysical results, such as negative turbulence kinetic energy, etc.

For constructing turbulence models, various model constraints have been proposed by different authors in an attempt to make the model equations as general (or universal) as possible. Most recent of these are, the realizability principle (Schumann 1977, Lumley 1978), the linearity principle (Pope 1983), the rapid distortion theory (Reynolds 1987) and the material indifference principle (Speziale 1983). However, some of the above mentioned principles are not universal. For example, the material indifference principle is not valid for general turbulence in which the fluctuating velocities are three dimensional. The material indifference is valid only for strict $2 \mathrm{D}-2 \mathrm{C}$ (two dimensional and two velocity component) turbulence which rarely occurs or probably does not exist in nature which is described by three dimensional space. Speziale et al. (1987), in their DNS experiments, found that rapid rotation had no influence on the isotropy of the 3D-3C homogeneous isotropic turbulence. So far, there is no evidence which shows a 3D-3C fluctuating velocity field will evolve to a strict $2 \mathrm{D}-2 \mathrm{C}$ state under any conditions. Therefore, the material indifference (or frame indifference) principle need not be considered in 3D-3C turbulence modeling. In addition, as we will see later, for strictly $2 \mathrm{D}-2 \mathrm{C}$ turbulence the frame indifference principle principle will be automatically satisfied and does not provide any additional or new constraints for turbulence models. In another study, Reynolds (1987) also showed that in 3D-3C turbulence modeling, at the $2 \mathrm{D}-2 \mathrm{C}$ limit, the frame indifference principle does not provide any new model constraints and it also seems to be irrelevant to the modeling of the effect of the rotation on turbulence (see the RDT described later). 
The principle of linearity is also not universal, as it holds only for passive scalars. It was pointed out by Pope (1983) that since the governing equations for passive scalars are linear, the modeled equations for these quantities should possess the same property. However, some of the models for the pressure correlations are nonlinear. The calculations of slightly heated turbulent jets (in which the temperature is a passive scalar) with such models (Shih et al 1990) show that the violation of this principle does not cause problems since the results agreed well with the experimental data (also see Lumley 1983).

Rapid distortion theory (RDT) is a convenient tool for studying the asymptotic behavior of turbulence under the rapid distortion of the mean flow. For example, effect of the mean flow (say strain rate) on turbulence. For some simple homogeneous flows RDT can provide an analytical solution (Reynolds 1987), hence enabling us to calculate the model terms of interest in the second moment equations. One may naturally ask if we can use these results to calibrate the model coefficients appearing in the corresponding model terms. Unfortunately, the answer is not very positive. RDT equations are valid only for very high mean strain rate or very low turbulent intensity and in both these cases the turbulence is in a very non-equilibrium state with the mean flow field. On the other hand the second order modeling is based on the assumption that the turbulence is more or less in an equilibrium state and is mainly characterized by one velocity and one length scale (Lumley 1983). Therefore, RDT results are probably not appropriate for tuning the coefficients of second order models. An interesting illustration of this point is made by Shih et al. (1990) who showed that a model constant in the linear form of rapid pressure strain model (Launder et al 1975) determined by matching RDT results is much worse than the one determined from measurements. However this does not mean that RDT is useless in turbulence modeling. On the contrary, RDT can provide the asymptotic behavior of turbulence at the RDT limit. In many cases, we may obtain analytical expressions which explicitly show the relations between turbulence and the mean flow field. For example, the RDT results (Reynolds 1989) for pure mean rotation show a clear effect of the mean rotation on the anisotropy of turbulence $\left(b_{i j}=\overline{u_{i} u_{j}} / q^{2}-\delta_{i j} / 3\right)$ through its second invariant. However, all the existing rapid pressure strain models (including the one which satisfies the material indifference at the $2 \mathrm{D}-2 \mathrm{C}$ limit) fail to exhibit any effect of rotation on the second invariant of $b_{i j}$. In this case, RDT will certainly guide us in removing this deficiency from the existing models.

Realizability (defined as the requirement of non-negative energy and Schwarz' inequality between any fluctuating quantities) is the basic physical and mathematical principle that any governing equation should obey. Hence, among all the above mentioned model constraints, realizability is the most universal, important and also the minimal requirement for the turbulence model equations to prevent them from producing unphysical results. Realizability can be applied to various turbulence quantities, for example, Reynolds stresses $\overline{u_{i} u_{j}}$, scalar fluxes $\overline{u_{i} \theta}$ and the triple correlations. For one-point second moment equations, realizability for $\overline{u_{i} u_{j}}$ and $\overline{u_{i} \theta}$ is the most important. In this paper (section 3, also see Shih and Lumley 1985) we will describe the principle of realizability, derive the realizability conditions for various model terms, and propose the model forms for pressure correlation 
terms in the second moment equations. The comparisons of various turbulence models with DNS and measurements will be presented in section 4. However, first we will discuss the $2 \mathrm{D}-2 \mathrm{C}$ turbulence.

\section{Two-Dimensional Two-Component Turbulence}

Reynolds and Grau (1988) argued that if one component of velocity is suppressed and turbulence becomes $2 \mathrm{C}$, the turbulence will not stay in the $2 \mathrm{C}$ state unless the turbulence also becomes two dimensional (2D). In the DNS experiment, Speziale et al (1987) were expecting that the homogeneous isotropic turbulence would become anisotropic under the action of rapid rotation. However, their results did not show any such trend. Can a wall bounded inhomogeneous turbulence become strictly 2D-2C turbulence under rapid rotation? In a three dimensional space, it seems unlikely that the instantaneous turbulent velocity field will become strictly or exactly $2 \mathrm{D}-2 \mathrm{C}$ turbulence. However, if we confine a flow to a two dimensional space, as in the "thin soap sheet film", then it may be possible to obtain a $2 \mathrm{D}-2 \mathrm{C}$ turbulence. Numerically it is always possible to simulate such a special "turbulence".

In this section, we will derive a $2 \mathrm{D}-2 \mathrm{C}$ turbulence model for the rapid pressure correlation term in the Reynolds stress equations (also see Reynolds 1987) and will show that the frame indifference principle does not provide any model constraints.

The Reynolds stress equation can be written as

$$
\frac{D}{D t} \overline{u_{i} u_{q}}=\ldots+T_{i q}-2 \nu \overline{u_{i, k} u_{q, k}}
$$

where

$$
\frac{T_{i q}}{\overline{q^{2}}} \equiv 2 U_{p, j}\left[M_{i j p q}+M_{q j p i}\right]
$$

corresponds to the rapid part of pressure correlation term, and $\overline{q^{2}}=\overline{u_{i} u_{i}}$. For homogeneous turbulence, $M_{i j p q}$ can be expressed as

$$
M_{i j p q}=\frac{1}{q^{2}} \int_{K} \frac{k_{p} k_{q}}{k^{2}} E_{i j}(\mathbf{k}) d^{2} \mathbf{k}
$$

where $k_{i}$ is the wave number vector and $E_{i j}$ is the spectrum tensor.

As usual, we assume that $M_{i j p q}$ is a function of the anisotropic tensor $b_{i j}$, and then the most general tensor form of $M_{i j p q}$ (which satisfies the symmetry in indices) will be 


$$
\begin{aligned}
M_{i j p q} & =a_{1} \delta_{i j} \delta_{p q}+a_{2}\left(\delta_{i p} \delta_{j q}+\delta_{i q} \delta_{j p}\right)+a_{3} \delta_{i j} b_{p q}+a_{4} \delta_{p q} b_{i j} \\
& +a_{5}\left(\delta_{i p} b_{j q}+\delta_{i q} b_{j p}+\delta_{j p} b_{i q}+\delta_{j q} b_{i p}\right) \\
& +a_{6} b_{i j} b_{p q}+a_{7}\left(b_{i p} b_{j q}+b_{i q} b_{j p}\right)
\end{aligned}
$$

where,

$$
\begin{aligned}
& b_{i j}=\frac{\overline{u_{i} u_{j}}}{q^{2}}-\frac{1}{2} \delta_{i j} \\
& \delta_{i i}=2 \\
& I I=-b_{i j} b_{i j} / 2
\end{aligned}
$$

Because of the Cayley-Hamilton theorem in a $2 \mathrm{D}$ space, $b_{i j}^{2}+I I \delta_{i j}=0$, the higher powers of $b_{i j}$ are not required. Seven coefficients $a_{i}$ are, in general, function of the invariants of $b_{i j}$. From the continuity and definition of $M_{i j p q}$, we have

$$
\begin{aligned}
M_{p j p q} & =0 \\
M_{i j p p} & =b_{i j}+\delta_{i j} / 2
\end{aligned}
$$

These give us the following relations between the coefficients:

$$
\begin{aligned}
& a_{1}+3 a_{2}-I I a_{6}-I I a_{7}=0 \\
& a_{3}+a_{4}+4 a_{5}=0 \\
& 2 a_{1}+2 a_{2}-2 I I a_{7}=1 / 2 \\
& 2 a_{4}+4 a_{5}=1
\end{aligned}
$$

The coefficients $a_{1}, a_{2}, a_{3}$ and $a_{4}$ can be solved in terms of $a_{5}, a_{6}$, and $a_{7}$ :

$$
\begin{aligned}
& a_{1}=3 / 8+I I\left(a_{7}-a_{6} / 2\right) \\
& a_{2}=-1 / 8+I I a_{6} / 2 \\
& a_{3}=-1 / 2-2 a_{5} \\
& a_{4}=1 / 2-2 a_{5}
\end{aligned}
$$

Therefore, the tensor $M_{i j p q}$ becomes

$$
\begin{aligned}
M_{i j p q} & =\frac{1}{8}\left(3 \delta_{i j} \delta_{p q}-\delta_{i p} \delta_{j q}-\delta_{i q} \delta_{j p}\right)+\frac{1}{2}\left(\delta_{p q} b_{i j}-\delta_{i j} b_{p q}\right) \\
& +a_{6}\left[b_{i j} b_{p q}-I I \delta_{i j} \delta_{p q} / 2+I I\left(\delta_{i p} \delta_{j q}+\delta_{i q} \delta_{j p}\right) / 2\right] \\
& +a_{7}\left(b_{i p} b_{j q}+b_{i q} b_{j p}+I I \delta_{i j} \delta_{p q}\right)
\end{aligned}
$$

where $a_{5}$ drops out because of the following zero tensor:

$$
\delta_{i p} b_{j q}+\delta_{i q} b_{j p}+\delta_{j p} b_{i q}+\delta_{j q} b_{i p}-2 \delta_{i j} b_{p q}-2 \delta_{p q} b_{i j} \equiv 0
$$


Using (2.9), the Eq.(2.2) becomes

$$
\frac{T_{i q}}{\overline{q^{2}}}=S_{i q}+4\left(a_{6}+2 a_{7}\right) b_{i j} b_{p q} S_{p j}+2 \Omega_{q j} b_{i j}+2 \Omega_{i j} b_{j q}
$$

where we have used the following relation:

$$
4 I I S_{i q}+\left[4 b_{i q} b_{j p}-2\left(b_{i j} b_{p q}+b_{i p} b_{j q}\right)\right] S_{p j}=0
$$

Equations (2.10) and (2.12) can be easily checked in the principal axes.

For the 2D-2C turbulence, the turbulence equation should be frame indifferent (Speziale 1983). If we write Eq.(2.1) in a non-inertial coordinate system (designated by ${ }^{*}$ ) which is rotating with an angular velocity $\Omega_{k}$, we obtain

$$
\begin{aligned}
\frac{D}{D t^{*}}{\overline{u_{i} u_{q}}}^{*} & =2\left(U_{p, j}\right)^{*}\left[M_{i j p q}+M_{q j p i}\right]^{*}{\overline{q^{2}}}^{*}+\nu \overline{u_{i, k} u_{q, k}}{ }^{*} \cdots \\
& -2 \varepsilon_{j i k} \Omega_{k}{\overline{u_{j} u_{q}}}^{*}-2 \varepsilon_{j q k} \Omega_{k}{\overline{u_{j} u_{i}}}^{*} \\
& -\varepsilon_{p j k} \Omega_{k}\left[M_{i j p q}+M_{q j p i}\right]^{*}{\overline{q^{2}}}^{*}
\end{aligned}
$$

In order for this equation to be frame-indifferent, the last two lines must cancel each other. This gives us the necessary constraint on the model form of $M_{i j p q}$ and the result is

$$
a_{4}-a_{3}=1
$$

However, this condition is already satisfied by the coefficients (2.8) and hence the frame indifference principle does not provide any additional constraints. Therefore, in general there is still one undetermined coefficient $a_{6}+2 a_{7}$ in (2.11) and it must be determined from experiment, direct numerical simulation or some other source. However, we may explore its limiting value by using $1 \mathrm{C}$ condition. The condition of $M_{i j p q}$ at the limit of $u_{1}=0$ gives $M_{1 j p q}=0$ for any $j, p$ and $q$ (because $E_{1 j}=0$ ). This condition gives

$$
a_{6}+2 a_{7}=-1 \quad \text { if } \quad b_{11}=-1 / 2, \quad\left(\text { or } u_{1}=0\right)
$$

However, we must note that in general 2D-2C flows, the value of $a_{6}+2 a_{7}$ is unknown and not necessarily -1 . The limiting value of the above coefficient can also be obtained by using the realizability conditions $(3.4 \mathrm{a}, \mathrm{b}, \mathrm{c})$ described in the next section. Interestingly enough such an analysis also gives $a_{6}+2 a_{7}=-1$.

\section{Three-Dimensional Three-Component Turbulence.}

It is commonly thought that the turbulence in a three dimensional space is always three dimensional (Tennekes and Lumley 1972) with three components of velocity. However, there are situations where the turbulence is considerably suppressed in a certain 
direction. For example when turbulence approaches a solid wall, the fluctuating velocity component normal to the wall will be mostly suppressed and the turbulence will become 3D-2C. However, during this process, all the turbulent energy components should remain non-negative and Schwarz' inequality should always be satisfied. This is the concept of realizability. In this section, we will discuss realizability in detail and discuss the model development of pressure correlation terms in the second moment equations.

\subsection{Realizability Concept.}

In the second order closure, model equations for the turbulent stresses $\overline{u_{i} u_{j}}$ and scalar fluxes $\overline{\theta u_{i}}$ are proposed. Realizability for both of these quantities has been discussed in Lumley (1978). To see the realizability concept, let us consider any two fluctuating variables $a$ and $b$, and their correlation matrix:

$$
\left(\begin{array}{ll}
\overline{a^{2}} & \overline{a b} \\
a b & \overline{b^{2}}
\end{array}\right)
$$

The simple fact that $\overline{a^{2}} \geq 0, \overline{b^{2}} \geq 0$, and that Schwarz inequality $\overline{a^{2}}{\overline{b^{2}}}_{\bar{a}^{2}} \overline{a b}^{2} \geq 0$ should be satisfied by any set of equations governing the evolution of $\overline{a^{2}}, \overline{b^{2}}$, and $\overline{a b}$ is called realizability. A set of equations violating this principle could produce unphysical results. We will show that these three realizability constraints translate to the single requirement that the eigenvalues of the correlation matrix should remain non-negative. We will now show that if any one of the above three in-equalities becomes an equality, then one of the eigenvalues of the matrix will vanish. Therefore, by ensuring these eigenvalues from becoming negative will automatically ensure $\overline{a^{2}}, \overline{b^{2}},\left(\overline{a^{2}} \frac{b^{2}}{-a b^{2}}\right)$ from becoming negative. This implies that while constructing governing equations for $a, b$ and their correlations, we need to check only the eigenvalues of the correlation matrix. The non-negativity of the eigenvalues of the matrix can be easily seen from their expression:

$$
\lambda_{1,2}=(1 / 2)\left\{\left(\overline{a^{2}}+\overline{b^{2}}\right) \pm\left[\left(\overline{a^{2}}+\overline{b^{2}}\right)^{2}-4\left(\overline{a^{2}} \overline{b^{2}}-\overline{a b}^{2}\right)\right]^{1 / 2}\right\}
$$

Eq. (3.1) shows that $\lambda_{2}$ will vanish if one of the following conditions occurs:

$$
\begin{aligned}
\overline{a^{2}} & =0, \\
\overline{b^{2}} & =0, \\
\overline{a^{2}} \overline{b^{2}}-\overline{a b}^{2} & =0 .
\end{aligned}
$$

Now, let us take the derivative of (3.1) with respect to time $t$,

$$
\begin{aligned}
& \left(\lambda_{1,2}\right)_{, t}=(1 / 2)\left\{\left(\overline{a^{2}}, t+\overline{b^{2}}, t\right)\right. \\
& \left. \pm \frac{\left(\overline{a^{2}}+\overline{b^{2}}\right)\left(\overline{a^{2}}, t+\overline{b^{2}}, t\right)-2\left[\left(\overline{a^{2}}\right)_{, t} \overline{b^{2}}+\overline{a^{2}}\left(\overline{b^{2}}\right)_{, t}-2 \overline{a b}(\overline{a b})_{, t}\right]}{\left[\left(\overline{a^{2}}+\overline{b^{2}}\right)^{2}-4\left(\overline{a^{2}} \overline{b^{2}}-\overline{a b}^{2}\right)\right]^{1 / 2}}\right\}
\end{aligned}
$$


To preserve the positive $\lambda_{2}$, we must require

$$
\left(\lambda_{2}\right)_{t} \geq 0, \quad \text { if } \quad \lambda_{2}=0
$$

or

$$
\frac{\left[\overline{a^{2}} \overline{b^{2}}-\overline{a b}^{2}\right]_{, t}}{\overline{a^{2}}+\bar{b}^{2}} \geq 0, \quad \text { if } \quad \lambda_{2}=0
$$

From this necessary condition, it is straight forward to show that

$$
\begin{aligned}
\overline{a^{2}}, t \geq 0, \quad \text { if } \quad \overline{a^{2}}=0 \\
\bar{b}^{2}, t \geq 0, \quad \text { if } \overline{b^{2}}=0 \\
\left(\overline{a^{2}} \overline{b^{2}}-\overline{a b}^{2}\right)_{, t} \geq 0, \quad \text { if } \overline{a^{2}} \overline{b^{2}}-\overline{a b}^{2}=0
\end{aligned}
$$

It is clear that (3.4) is the necessary condition for the quantities $\overline{a^{2}}, \overline{b^{2}}$ and $\overline{a^{2}} \overline{b^{2}}-$ $\overline{a b}^{2}$ to remain non-negative, therefore, the condition (3.3a) is the necessary condition for realizability. To find the sufficient condition for realizability, we must examine the higher order derivative of the eigenvalue when it vanishes. For example, we may require

$$
\left(\lambda_{2}\right)_{, t t} \geq 0, \quad \text { if } \quad \lambda_{2}=0 .
$$

For more details on this aspect see the review paper by Lumley (1983).

Similar arguments can be applied to the turbulent stress tensor $\overline{u_{i} u_{j}}$ and to the correlation matrix formed with a scalar $\theta$ and velocities $u_{i}$. Realizability for tensor $\overline{u_{i} u_{j}}$ is straight forward, that is, its eigenvalues must be positive, which will ensure positive turbulent energy components and the Schwarz inequality between fluctuating velocities. Realizability for the correlation matrix of scalar and velocity is described by Eq. (3.1) and (3.3) and is not as simple as for the Reynolds stress. Lumley has suggested a more general quantity $D_{i j}$ consisting of turbulent stress and flux: $D_{i j}=\overline{\theta^{2}} \overline{u_{i} u_{j}}-\overline{\theta u_{i}} \overline{\theta u_{j}}$. It can be shown that realizability for the correlation matrix between the velocity and the scalar will be ensured provided that the eigenvalues of $D_{i j}$ are positive.

It is interesting to note that if $u_{\alpha} \rightarrow 0$ then the $\alpha$ direction will become one of the principal axes of $\overline{u_{i} u_{j}}$, and $\overline{u_{\alpha} u_{\alpha}}$ (no summation on Greek indices) becomes one of the eigenvalues of $\overline{u_{i} u_{j}}$. At the same time, $u_{\alpha} \rightarrow 0$ will make $D_{\alpha \alpha} \rightarrow 0$, and the $\alpha$ direction also becomes one of the principal axes of $D_{i j} . D_{\alpha \alpha}$ is then a vanishing eigenvalue of $D_{i j}$. However $D_{\alpha \alpha}$ will also vanish without $u_{\alpha} \rightarrow 0$ if the perfect correlation between $f$ and $u_{\alpha}$ is reached. Therefore, we conclude that if one of the eigenvalues of $\overline{u_{i} u_{j}}$, say $\overline{u_{\alpha} u_{\alpha}}$, vanishes, then $D_{\alpha \alpha}$ will become a vanishing eigenvalue of the tensor $D_{i j}$. However, the reverse is not true i.e. if $D_{\alpha \alpha} \rightarrow 0$, it does not mean that $\overline{u_{\alpha} u_{\alpha}}$ (or $\overline{\theta^{2}}$ ) must vanish. This is important in the modeling of scalar turbulence, as it indicates that realizability based on $\overline{u_{i} u_{j}}$ alone is not enough and we must also consider the joint realizability based on $D_{i j}$ to ensure that the model equations do not produce unphysical results. 


\subsection{Realizability Conditions for Second Order Closure Models}

The ideas discussed above can now be applied to the second moment equations to obtain the realizability conditions. The general form of the model equations for the second moments can be written as follows

$$
\begin{aligned}
\frac{D}{D t} \overline{u_{i} u_{j}}= & -\left[\overline{u_{i} u_{j} u_{k}}-C\left(\overline{q^{2} u_{i}} \delta_{j k}+\overline{q^{2} u_{j}} \delta_{i k}\right)\right]_{, k} \\
& -\left(\overline{u_{i} u_{k}} U_{j, k}+\overline{u_{j} u_{k}} U_{i, k}\right)+2 U_{p, q}\left(X_{p j q i}+X_{p i q j}\right) \\
& -\beta_{k}\left(Y_{k i j}+Y_{k j i}\right)-\left(\Phi_{i j}+2 \delta_{i j} / 3\right) \epsilon \\
& +\beta_{i} \overline{\theta u_{j}}+\beta_{j} \overline{\theta u_{i}} \\
\frac{D}{D t} \overline{\theta u_{i}}= & -\left[\overline{\theta u_{i} u_{k}}-C^{\prime} \overline{\theta q^{2}} \delta_{i k}\right]_{, k}-\left(\overline{\theta u_{k}} U_{i, k}+\overline{u_{i} u_{k}} \Theta_{, k}\right)+\beta_{i} \overline{\theta^{2}} \\
& +2 U_{j, k} X_{i j k}-\beta_{k} Y_{i k}-\Phi_{i} \epsilon \overline{q^{2}} \\
\frac{D}{D t} \overline{\theta^{2}}= & -\left(\overline{\theta^{2} u_{k}}\right)_{, k}-2 \overline{\theta u_{k}} \Theta_{, k}-2 \epsilon_{\theta}
\end{aligned}
$$

where we have followed Lumley (1978) for the pressure transport model and in combining the anisotropic part of the dissipation terms with the slow part of the pressure correlations. The molecular diffusion terms in the above equations have been neglected. The definitions of the rapid pressure correlation terms $X_{p j q i}$ and $X_{i j k}$; the buoyancy terms $Y_{k i j}$ and $Y_{i k}$; and the slow terms $\Phi_{i j}$ and $\Phi_{i}$ are listed in appendix $A$. In the above equations $\beta_{i}$ is the buoyancy vector, $\Theta$ is the mean scalar and $\theta$ is the fluctuating scalar.

To impose realizability into the above equations we may write the equations of $\overline{u_{i} u_{j}}$ and $D_{i j}$ in their principal axes, i.e., the equations for $\overline{u_{\alpha} u_{\alpha}}$ and $D_{\alpha \alpha}$. Then in order to ensure that the eigenvalues of $\overline{u_{\alpha} u_{\alpha}}$ and $D_{\alpha \alpha}$ remain positive during the evolution of turbulence, the following constraints must be satisfied

$$
\begin{aligned}
& \frac{D}{D t} \overline{u_{\alpha} u_{\alpha}} \rightarrow 0 \text { if } \overline{u_{\alpha} u_{\alpha}} \rightarrow 0 \\
& \frac{D}{D t} D_{\alpha \alpha} \rightarrow 0 \text { if } \quad D_{\alpha \alpha} \rightarrow 0 \\
& \frac{D^{2}}{D t^{2}} \overline{u_{\alpha} u_{\alpha}} \geq 0 \text { if } \overline{u_{\alpha} u_{\alpha}} \rightarrow 0 \\
& \frac{D^{2}}{D t^{2}} D_{\alpha \alpha} \geq 0 \text { if } \quad D_{\alpha \alpha} \rightarrow 0
\end{aligned}
$$

Substituting (3.6)-(3.8) into (3.9) we obtain the following necessary realizability conditions for $X_{p j q i}, X_{i j k}, Y_{i k j}$ and $Y_{i k}$ :

$$
\begin{aligned}
& U_{p, q} X_{p \alpha q \alpha} \\
& \text { if } \overline{u_{\alpha}^{2}} \rightarrow 0
\end{aligned}
$$




$$
\begin{aligned}
& U_{p, q}\left(\overline{\theta^{2}} X_{p \alpha q \alpha}-\overline{\theta u_{\alpha}} X_{\alpha p q}\right) \quad \rightarrow 0 \\
& \text { if } \left.D_{\alpha \alpha} \rightarrow 0\left(\overline{u_{\alpha}^{2}} \neq 0\right) \quad \text { (in p.a. of } D_{i j}\right) \\
& U_{p, q} \overline{\theta u_{\alpha}} X_{\alpha p q} \quad \rightarrow 0 \\
& \text { if } D_{\alpha \alpha}, \overline{u_{\alpha}^{2}} \rightarrow 0 \quad \text { (in p.a. of } D_{i j}, R_{i j} \text { ) } \\
& Y_{\alpha \alpha \alpha}-\overline{\theta u_{\alpha}} \quad \rightarrow 0 \\
& \text { if } D_{\alpha \alpha}, \overline{u_{\alpha}^{2}} \rightarrow 0 \quad \text { (in p.a. of } D_{i j}, R_{i j} \text { ) } \\
& Y_{\gamma \alpha \alpha}(\gamma \neq \alpha) \quad \rightarrow 0 \\
& \text { if } D_{\alpha \alpha}, \overline{u_{\alpha}^{2}} \rightarrow 0 \quad \text { (in p.a. of } D_{i j}, R_{i j} \text { ) } \\
& \overline{\theta^{2}} Y_{k \alpha \alpha}-\overline{\theta u_{\alpha}} Y_{k \alpha}(\text { any } k) \quad \rightarrow 0 \\
& \text { if } \left.D_{\alpha \alpha} \rightarrow 0\left(\overline{u_{\alpha}^{2}} \neq 0\right) \quad \text { (in p.a. of } D_{i j}\right) \\
& \overline{\theta u_{\alpha}} Y_{\alpha \alpha}-\overline{\theta^{2}} \overline{\theta u_{\alpha}} \quad \rightarrow 0 \\
& \text { if } D_{\alpha \alpha}, \overline{u_{\alpha}^{2}} \rightarrow 0 \quad \text { (in p.a. of } D_{i j}, R_{i j} \text { ) } \\
& \overline{\theta u_{\alpha}} Y_{\gamma \alpha}(\gamma \neq \alpha) \quad \rightarrow 0 \\
& \text { if } D_{\alpha \alpha}, \overline{u_{\alpha}^{2}} \rightarrow 0 \quad \text { (in p.a. of } D_{i j}, R_{i j} \text { ) } \\
& \left(\Phi_{\alpha \alpha}+2 / 3\right) \epsilon \quad \rightarrow F^{a} \\
& \text { if } \overline{u_{\alpha}^{2}} \rightarrow 0 \quad \text { (in p.a. of } R_{i j} \text { ) } \\
& 2 \overline{\theta u_{\alpha}} \Phi_{\alpha} \epsilon / \overline{q^{2}}-\overline{\theta^{2}}\left(\Phi_{\alpha \alpha}+2 / 3\right) \epsilon-2 \epsilon_{\theta} \overline{u_{\alpha}^{2}} \quad \rightarrow F_{d}^{b} \\
& \text { if } D_{\alpha \alpha} \rightarrow 0\left(\overline{u_{\alpha}^{2}} \neq 0\right) \text { (in p.a. of } D_{i j} \text { ) } \\
& 2 \overline{\theta u_{\alpha}} \Phi_{\alpha} \epsilon / \overline{q^{2}}-2 \epsilon_{\theta} \overline{u_{\alpha}^{2}}+\overline{\theta^{2}} F^{a} \rightarrow F_{d}^{b} \\
& \text { if } D_{\alpha \alpha}, \overline{u_{\alpha}^{2}} \rightarrow 0 \quad \text { (in p.a. of } D_{i j}, R_{i j} \text { ) } \\
& \left.\overline{\left(\overline{u_{\alpha}^{2} u_{k}}\right.}-2 C \overline{q^{2} u_{\alpha}} \delta_{\alpha k}\right)_{, k} \rightarrow 0 \\
& \text { if } \overline{u_{\alpha}^{2}} \rightarrow 0 \quad \text { (in p.a. of } R_{i j} \text { ) } \\
& 2 \overline{\theta u_{\alpha}}\left(\overline{\theta u_{\alpha} u_{k}}-C^{\prime} \overline{q^{2} \theta} \delta_{\alpha k}\right)_{, k} \\
& -\overline{\theta^{2}}\left(\overline{u_{\alpha}^{2} u_{k}}-2 C \overline{q^{2} u_{\alpha}} \delta_{\alpha k}\right)_{, k}-\overline{u_{\alpha}^{2}}\left(\overline{\theta u_{k}}\right)_{, k} \quad \rightarrow 0
\end{aligned}
$$




$$
\begin{aligned}
& \text { if } D_{\alpha \alpha} \rightarrow 0\left(\overline{u_{\alpha}^{2}} \neq 0\right) \quad\left(\text { in p.a. of } D_{i j}\right) \\
& 2 \overline{\theta u_{\alpha}}\left(\overline{\theta u_{\alpha} u_{k}}-C^{\prime} \overline{q^{2} \theta} \delta_{\alpha k}\right)_{, k}-\overline{u_{\alpha}^{2}}\left(\overline{\theta u_{k}}\right)_{, k} \quad \rightarrow 0 \\
& \text { if } D_{\alpha \alpha}, \overline{u_{\alpha}^{2}} \rightarrow 0 \quad\left(\text { in p.a. of } D_{i j}, R_{i j}\right)
\end{aligned}
$$

These constraints will be used in the next section in constructing the models for pressure-correlations.

\subsection{Modeling of the Rapid Pressure-Correlation Terms}

First let us look at the rapid terms associated with the mean strain. The symmetry property of these terms requires

$$
X_{p j q i}=X_{j p q i}, \quad X_{p j q i}=X_{p j i q}, \quad X_{i j k}=X_{j i k}
$$

If we assume that $X_{p j q i}$ is a function of $\overline{u_{i} u_{j}}$ only and that $X_{i j k}$ is a function of both $\overline{u_{i} u_{j}}$ and $\overline{\theta u_{i}}$, but linear in $\overline{\theta u_{i}}$, then the most general forms of $X_{p j q i}$ and $X_{i j k}$ satisfying the above symmetry constraints are

$$
\begin{aligned}
X_{p j q i} / \overline{q^{2}} & =\alpha_{1} \delta_{q i} \delta_{p j}+\alpha_{2}\left(\delta_{p q} \delta_{i j}+\delta_{q j} \delta_{p i}\right)+\alpha_{3} \delta_{q i} b_{p j}+\alpha_{4} \delta_{p j} b_{q i} \\
& +\alpha_{5}\left(\delta_{p q} b_{i j}+\delta_{i j} b_{p q}+\delta_{j q} b_{p i}+\delta_{p i} b_{q j}\right)+\alpha_{6} \delta_{q i} b_{p j}^{2}+\alpha_{7} \delta_{p j} b_{q i}^{2} \\
& +\alpha_{8}\left(\delta_{p q} b_{i j}^{2}+\delta_{i j} b_{p q}^{2}+\delta_{j q} b_{p i}^{2}+\delta_{p i} b_{q j}^{2}\right)+\alpha_{9} b_{q i} b_{p j} \\
& +\alpha_{10}\left(b_{p q} b_{i j}+b_{q j} b_{p i}\right)+\alpha_{11} b_{q i} b_{p j}^{2}+\alpha_{12} b_{p j} b_{q i}^{2} \\
& +\alpha_{13}\left(b_{p q} b_{i j}^{2}+b_{i j} b_{p q}^{2}+b_{q j} b_{p i}^{2}+b_{p i} b_{q j}^{2}\right) \\
& +\alpha_{14} b_{q i}^{2} b_{p j}^{2}+\alpha_{15}\left(b_{p q}^{2} b_{i j}^{2}+b_{q j}^{2} b_{p i}^{2}\right) \\
X_{i k j} & =\beta_{1} \delta_{i k} \overline{\theta u_{j}}+\beta_{2}\left(\delta_{i j} \overline{\theta u_{k}}+\delta_{j k} \overline{\theta u_{i}}\right)+\beta_{3} b_{i k} \overline{\theta u_{j}} \\
& +\beta_{4}\left(b_{i j} \overline{\theta u_{k}}+b_{j k} \overline{\theta u_{i}}\right)+\beta_{5}\left(\delta_{i j} b_{k p}+\delta_{k j} b_{i p}\right) \overline{\theta u_{p}} \\
& +\beta_{6} \delta_{i k} b_{j p} \overline{\theta u_{p}}+\beta_{7} b_{i k} b_{j p} \overline{\theta u_{p}}+\beta_{8}\left(b_{i j} b_{k p}+b_{k j} b_{i p}\right) \overline{\theta u_{p}} \\
& +\beta_{9} b_{i k}^{2} \overline{\theta u_{j}}+\beta_{10}\left(b_{i j}^{2} \overline{\theta u_{k}}+b_{j k}^{2} \overline{\theta u_{i}}\right)+\beta_{11} \delta_{i k} b_{j p}^{2} \overline{\theta u_{p}} \\
& +\beta_{12}\left(\delta_{i j} b_{k p}^{2}+\delta_{j k} b_{i p}^{2}\right) \overline{\theta u_{p}}+\beta_{13} b_{i k}^{2} b_{j p} \overline{\theta u_{p}}+\beta_{14} b_{i k} b_{j p}^{2} \overline{\theta u_{p}} \\
& +\beta_{15}\left(b_{i j}^{2} b_{k p}+b_{k j}^{2} b_{i p}\right) \overline{\theta u_{p}}+\beta_{16}\left(b_{i j} b_{k p}^{2}+b_{k j} b_{i p}^{2}\right) \overline{\theta u_{p}} \\
& +\beta_{17} b_{i k}^{2} b_{j p}^{2} \overline{\theta u_{p}}+\beta_{18}\left(b_{i j}^{2} b_{k p}^{2}+b_{k j}^{2} b_{i p}^{2}\right) \overline{\theta u_{p}}
\end{aligned}
$$

Where $\alpha$ 's are, in general, functions of the second and third invariants (i.e. II and III) of the anisotropic tensor $b_{i j}$ and $\beta$ 's are functions of the invariants formed by $b_{i j}$ and $\overline{\theta u_{i}}$ 
in addition to II and III. From the definition of the rapid terms and incompressibility, following properties are obtained:

$$
X_{p p q i}=\overline{u_{q} u_{i}}, \quad X_{p k q k}=0, \quad X_{i i k}=\overline{\theta u_{k}}, \quad X_{i k k}=0
$$

Using these properties and the realizability conditions given in the last section we can determine the limiting values of all the model coefficients provided we ignore the terms with the fourth powers of $b_{i j}$ (which is a reasonable assumption since the magnitude of $b_{i j}$ is always less than unity). The final expressions of the $X_{p j q i}$ and $X_{i j k}$ reduce to the surprisingly simple forms and are given by

$$
\begin{aligned}
X_{p j q i} / \overline{q^{2}} & =\frac{1}{30}\left(4 \delta_{p j} \delta_{q i}-\delta_{p q} \delta_{i j}-\delta_{q j} \delta_{p i}\right)-\frac{1}{3}\left(\delta_{q i} b_{p j}-\delta_{p j} b_{q i}\right) \\
& +a_{1}\left(\delta_{p q} b_{i j}+\delta_{i j} b_{p q}+\delta_{q j} b_{p i}+\delta_{p i} b_{q j}-\frac{11}{3} \delta_{q i} b_{p j}-\frac{4}{3} \delta_{p j} b_{q i}\right) \\
& +a_{2}\left(2 \delta_{p j} b_{q i}^{2}-3 b_{p q} b_{i j}-3 b_{q j} b_{p i}+b_{q i} b_{p j}\right) \\
X_{i j k}= & \frac{2}{5} \delta_{i j} \overline{\theta u_{k}}-\frac{1}{10}\left(\delta_{i k} \overline{\theta u_{j}}+\delta_{j k} \overline{\theta u_{i}}\right) \\
& +C_{D 1} b_{i j} \overline{\theta u_{k}} \\
& +C_{D 2}\left(b_{i k} \overline{\theta u_{j}}+b_{j k} \bar{\theta} \overline{\theta u_{i}}\right)+C_{D 3} \delta_{i j} b_{k l} \overline{\theta u_{l}}
\end{aligned}
$$

where the limiting values of the coefficients are

$$
a_{1}=-\frac{1}{10}, a_{2}=\frac{1}{10}, C_{D 1}=\frac{1}{10}, C_{D 2}=-\frac{3}{10}, C_{D 3}=\frac{1}{5}
$$

The last line in both (3.15) and (3.16) represents the non-linear contribution, and if neglected, the linear models used by various other workers will be recovered. It is important to note that the above values of the coefficients $a_{1}, a_{2}, C_{D 1}, C_{D 2}$, and $C_{D 3}$ are their limiting values at the realizability limit, i.e. when $\overline{u_{\alpha} u_{\alpha}}, D_{\alpha \alpha} \rightarrow 0$. For general turbulent flows $\overline{u_{\alpha} u_{\alpha}}$ and $D_{\alpha \alpha}$ are not zero and hence the values of the coefficients may deviate from their limiting values. As was pointed out before, in gencral $a$ 's are functions of II and III, and $C_{D}$ 's are functions of the invariants formed by the anisotropic tensor and the heat flux. Some guidance can be obtained by inspecting the following two useful parameters (see Lumley 1978, and Shih and Lumley 1986)

$$
\begin{gathered}
F=1+27 I I I+9 I I \\
F_{d}=9 d_{i i}^{3}-\frac{27}{2} d_{i i}^{2}+\frac{9}{2}
\end{gathered}
$$

where

$$
\begin{gathered}
I I=-\frac{1}{2} b_{i i}^{2}, \quad I I I=\frac{1}{3} b_{i i}^{3} \\
d_{i j}=\frac{\overline{\theta^{2}} \overline{u_{i} u_{j}}-\overline{\theta u_{i}} \overline{\theta u_{j}}}{\overline{\theta^{2}} \overline{u_{i} u_{i}}-\overline{\theta u_{i}} \overline{\theta u_{i}}}
\end{gathered}
$$


It can be shown that both $F$ and $F_{d}$ are bounded between 0 and 1, and particularly

$$
\begin{aligned}
F & \rightarrow 0 \text { when } \overline{u_{\alpha} u_{\alpha}} \rightarrow 0 \\
F_{d} & \rightarrow 0 \text { when } D_{\alpha \alpha} \rightarrow 0
\end{aligned}
$$

By using this information, it is convenient to write

$$
\begin{aligned}
a_{1} & =-\frac{1}{10}\left(1+A F^{\alpha}\right) \\
a_{2} & =\frac{1}{10}\left(1+B F^{\alpha}\right) \\
C_{D 1} & =\frac{1}{10}+C_{1} F_{d}^{\alpha} \\
C_{D 2} & =\frac{-3}{10}+C_{2} F_{d}^{\alpha} \\
C_{D 3} & =\frac{1}{5}+C_{3} F_{d}^{\alpha}
\end{aligned}
$$

where $A, B, C_{1}, C_{2}$ and $C_{3}$ are adjustable constants but $\alpha$ is not as arbitrary as it might seem at first look. The condition (3.10) suggests $\alpha=\frac{1}{2}$ (see Lumley 1983, or Shih and Lumley 1986). In the limiting case these coefficients reach their limiting values. Shih et al $(1985,1987)$ took $A=0.8$ and $B=0.0$ which fit the DNS data quite well. However, it seems more judicious to use homogeneous shear flow experiment to obtain the values of these coefficients (this is not done in the present study). Shil et al (1990) set $C_{1}=$ $C_{2}=C_{3}=0$ in their computations. The present study indicates that the full form of $C_{D 1}, C_{D 2}$ and $C_{D 3}$ should be used. The experiment of Tavalouris and Corrsin (1981) suggests $C_{1}=1.8, C_{2}=-1.8$, and $C_{3}=4.5$.

Now returning to the modeling of the buoyancy terms $Y_{k i j}$ and $Y_{i k}$, we assume that these are functions of the anisotropic tensor and the heat flux. Their minimum forms required to satisfy realizability are as follows

$$
\begin{aligned}
Y_{i k j} & =\beta_{1} \delta_{i k} \overline{\theta u_{j}}+\beta_{2}\left(\delta_{i j} \overline{\theta u_{k}}+\delta_{j k} \overline{\theta u_{i}}\right)+\beta_{3} b_{i k} \overline{\theta u_{j}} \\
& +\beta_{4}\left(b_{i j} \overline{\theta u_{k}}+b_{j k} \overline{\theta u_{i}}\right)+\beta_{5}\left(\delta_{i j} b_{k p}+\delta_{k j} b_{i p}\right) \overline{\theta u_{p}} \\
& +\beta_{6} \delta_{i k} b_{j p} \overline{\theta u_{p}}+\beta_{7} b_{i k} b_{j p} \overline{\theta u_{p}}+\beta_{8}\left(b_{i j} b_{k p}+b_{k j} b_{i p}\right) \overline{\theta u_{p}} \\
& +\beta_{9} b_{i k}^{2} \overline{\theta u_{j}}+\beta_{10}\left(b_{i j}^{2} \overline{\theta u_{k}}+b_{j k}^{2} \overline{\theta u_{i}}\right)+\beta_{11} \delta_{i k} b_{j p}^{2} \overline{\theta u_{p}} \\
& +\beta_{12}\left(\delta_{i j} b_{k p}^{2}+\delta_{j k} b_{i p}^{2}\right) \overline{\theta u_{p}} \\
Y_{i k} & =\gamma_{1} \overline{\theta^{2}} \delta_{i k}+\gamma_{2} \overline{\theta^{2}} b_{i k}+\gamma_{3} \overline{\theta u_{i}} \overline{\theta u_{k}}+\gamma_{4}\left(\overline{\theta u_{k}} b_{i p} \overline{\theta u_{p}}+\overline{\theta u_{i}} b_{k p} \overline{\theta u_{p}}\right) \\
& +\gamma_{5} \overline{\theta^{2}} b_{i k}^{2}+\gamma_{6} b_{i p} b_{k q} \overline{\theta u_{p}} \overline{\theta u_{q}} \\
& +\gamma_{7}\left(b_{i p}^{2} \overline{\theta u_{p}} \overline{\theta u_{K}}+b_{k p}^{2} \overline{\theta u_{p}} \overline{\theta u_{i}}\right)
\end{aligned}
$$

The above forms already satisfy the following symmetry conditions

$$
Y_{k i j}=Y_{i k j}, \quad Y_{i k}=Y_{k i}
$$


By definition, and incompressibility, these tensors have the following additional properties:

$$
Y_{k k j}=\overline{\theta u_{j}}, \quad Y_{k k}=\overline{\theta^{2}}
$$

Using these and the realizability conditions given in the last section, all except one coefficient can be determined exactly. Their final forms are

$$
\begin{aligned}
& \beta_{1}=\frac{4}{3}\left(1-\beta_{5}\right) \\
& \beta_{2}=\frac{\left(\beta_{5}-1\right)}{3} \\
& \beta_{3}=\frac{\left(1-11 \beta_{5}\right)}{3} \\
& \beta_{4}=\beta_{5}-1 \\
& \beta_{5}=\frac{-(12 I I+7) \underline{r^{2}}}{6 I I \overline{\theta^{2} q^{2}}-10 \underline{r^{2}}-36 I I \underline{r b r}} \\
& \beta_{6}=\frac{\left(2-4 \beta_{5}\right)}{3} \\
& \beta_{7}=\frac{-7}{6 I I}+\beta_{5}\left(\frac{5}{3 I I}-1\right) \\
& \beta_{8}=3 \beta_{5} \\
& \beta_{9}=\frac{7}{6 I I}-\beta_{5}\left(\frac{5}{3 I I}\right) \\
& \beta_{10}=0 \\
& \beta_{11}=-2 \beta_{5} \\
& \beta_{12}=0 \\
& \gamma_{1}=\frac{\left(6 \underline{r^{2}}+10 \overline{\theta^{2}} \overline{q^{2}}\right)}{9 \overline{\theta^{2}} \overline{q^{2}}} \\
& -\beta_{5} \frac{\left[18\left(\underline{r^{2}}+\overline{\theta^{2}} \overline{q^{2}}\right) \underline{r b r}+\underline{r^{2}}\left(7 \overline{\theta^{2}} \overline{q^{2}}-15 \underline{r^{2}}\right)+(36 I I-10)\left(\overline{\theta^{2}} \overline{q^{2}}\right)^{2}\right]}{9 \overline{\theta^{2}} \overline{q^{2}}\left(\underline{r^{2}}-\overline{\theta^{2}} \overline{q^{2}}\right)} \\
& +\gamma_{6} \frac{\left(\overline{q^{2}} / 9\right)\left(3 \underline{r b r}+6 I I \underline{r^{2}}-2 I I \overline{\theta^{2}} \overline{q^{2}}\right)}{\left(\underline{r^{2}}-\overline{\theta^{2}} \overline{q^{2}}\right)} \\
& \gamma_{2}=\frac{\underline{r}^{2}(6 I I+28)-\overline{\theta^{2}} \overline{q^{2}}(6 I I+7)}{18 I I\left(\underline{r^{2}}-\overline{\theta^{2}} \overline{q^{2}}\right)}-\frac{7\left(\underline{r^{2}}\right)^{2}}{(6 I I) \overline{\theta^{2}} \frac{\overline{q^{2}}}{\left(\underline{r^{2}}-\overline{\theta^{2}} \overline{q^{2}}\right)}} \\
& -\beta_{5}\left[\frac{\underline{r^{2}}(12 I I+20)+108 I I \underline{r b r}+\overline{\theta^{2}} \overline{q^{2}}\left(108 I I^{2}-21 I I-5\right)}{9 I I\left(\underline{r^{2}}-\overline{\theta^{2}} \overline{q^{2}}\right)}\right. \\
& \left.-\frac{5(3 I I+1)\left(\underline{r^{2}}\right)^{2}}{3 I I \overline{\theta^{2}} \overline{q^{2}}\left(\underline{r^{2}}-\overline{\theta^{2}} \overline{q^{2}}\right)}\right] \\
& +\gamma_{6}\left[\frac{\underline{r^{2}} \overline{q^{2}}(18 I I+4)+9 \overline{q^{2}} \underline{r b r}-\overline{\theta^{2}} \overline{q^{2}}(6 I I+1) \overline{q^{2}}}{9\left(\underline{r^{2}}-\overline{\theta^{2}} \overline{q^{2}}\right)}\right.
\end{aligned}
$$




$$
\begin{aligned}
& \left.-\frac{\underline{r^{2}}}{3 \theta^{2}} \frac{\underline{r^{2}}}{\left(\underline{r^{2}}-\overline{\theta^{2}} \overline{q^{2}}\right)}\right] \\
\gamma_{3}= & \frac{-2}{\overline{q^{2}}}+\beta_{5} \frac{12 \underline{r b r}-5 \underline{r^{2}}+(12 I I-1) \overline{\theta^{2}} \overline{q^{2}}}{\overline{q^{2}}\left(\underline{r^{2}}-\overline{\theta^{2}} \overline{q^{2}}\right)} \\
& -\gamma_{6} \frac{6 I \underline{r^{2}}+3 \underline{\underline{r b r}-2 I I \overline{\theta^{2}} \frac{q^{2}}{q^{2}}}}{3\left(\underline{r^{2}}-\overline{\theta^{2}} \overline{q^{2}}\right)} \\
\gamma_{4}= & \frac{-3 \beta_{5}}{\overline{q^{2}}} \\
\gamma_{5}= & \frac{7}{6 I I}+\beta_{5} \frac{(18 I I-5)}{3 I I}+\gamma_{6}\left(\frac{\overline{q^{2}}}{3}-\frac{r^{2}}{\overline{\overline{\theta^{2}}}}\right) \\
\gamma_{6}= & \text { undetermined coefficient } \\
\gamma_{7}= & 0
\end{aligned}
$$

where $\underline{r^{2}}=\overline{\theta u_{i}} \overline{\theta u_{i}}, \underline{r b r}=\overline{\theta u_{i}} b_{i j} \overline{\theta u_{j}}$. These expressions may seem a bit complicated but they ensure joint realizability. Since $\gamma_{6}$ is undetermined it was taken as zero in the calculations to be presented later in this paper. As an illustration, Appendix B lists the values of all the above coefficients as calculated from the buoyant plume experiment (1990).

\subsection{Slow Terms}

Lumley (1978) has suggested the following expression for the slow term of the pressurestrain correlation

$$
\Phi_{i j}=\beta b_{i j}+\gamma\left(b_{i k} b_{k j}+2 I I \delta_{i j} / 3\right)
$$

where $\beta$ and $\gamma$ are, in general, functions of the invariants II and III, and the Reynolds number. Most of the workers have used Rotta's hypothesis which is obtained by ignoring the nonlinear term. In LRR model, $\beta$ is taken as a constant, whereas Lumley (1978) proposed an expression involving II, III and the turbulence Reynolds number. This expression for $\beta$ was calibrated from experiments in which invariant III was negative. Gence and Mathieu (1980) and Choi (1983) experimentally studied the return to isotropy of homogeneous flows and found that the return to isotropy is slower when the invariant III is positive. Choi and Lumley (1984) suggested keeping the complete form of (3.27) and using their experiments proposed new forms of $\beta$ and $\gamma$ (see Appendix C). We will use both Lumley (1978) and Choi and Lumley (1984) models with the presently proposed models for the rapid term to see how the combinations fair.

For the slow part of the pressure temperature-gradient correlation Shih and Lumley (1985)proposed

$$
\Phi_{i}=f_{1} \overline{\theta u_{i}}+f_{2} b_{i k} \overline{\theta u_{k}}
$$

Again most workers ignore the second term and take $f_{1}$ as a constant. Shih and Lumley 
(1986) and Shih et al (1990) use a variable form of $f_{1}$ given by

$$
f_{1}=\frac{\beta}{2}+\frac{\epsilon_{\theta} \overline{q^{2}}}{\overline{\theta^{2}} \epsilon}-\frac{(\beta-2) I I_{d} / 6}{\left(I I_{d} / 3+b_{i j} d_{i j}^{2}-b_{i j} d_{i j}\right)}+H F_{d}^{1 / 2}
$$

and take $f_{2}$ as zero. The invariant $I I_{d}$ appearing in the above expression is defined by

$$
I I_{d}=\left(d_{i j} d_{i j}-d_{i i}^{2}\right) / 2
$$

This expression is based on the joint realizability principle and the parameter $H$ is given in Shih et al (1990) and $\beta$ is the same as proposed by Lumley (1978). Both of these functions are given in Appendix C. In the present study, we apply the joint realizability condition to (3.28) keeping the full non-linear model for the pressure strain to obtain

$$
\begin{aligned}
f_{1}= & \frac{\beta}{2}+\frac{\epsilon_{\theta} \overline{q^{2}}}{\overline{\theta^{2}} \epsilon}-\frac{(\beta+\gamma I I-2) I I_{d} / 6}{\left(I I_{d} / 3+b_{i j} d_{i j}^{2}-b_{i j} d_{i j}\right)} \\
& +\frac{\gamma}{2} \frac{\left(-I I I I_{d}+b_{i j}^{2} d_{i j}^{2}-b_{i j}^{2} d_{i j}\right)}{\left(I I_{d} / 3+b_{i j} d_{i j}^{2}-b_{i j} d_{i j}\right)}+H F_{d}^{1 / 2}
\end{aligned}
$$

where $\beta$ and $\gamma$ are the same coefficients as appearing in (3.27). For $\gamma=0$, the Shih and Lumley (1986) model is recovered. In the present study we used the $\beta$ and $\gamma$ as given by Choi and Lumley (1984) and these are reproduced in Appendix C.The above expression seems to give better agreement with the experiments. In the experiments analysed, the value of $f_{1}$ varied from $6.4-8.0$. The values used by Zeman and Lumley (1976), $f_{1}=7.5$, and Newman et al (1981), $f_{1}=6.6$, fall within this range.

\subsection{Other Models}

Other researchers, who employ the realizability concept, approach the models for pressure correlation terms in a similar but slightly different way. Craft et al. (1989) have proposed non-linear models for these correlations. They start with the similar form of the models as Shih and Lumley (1985), but apply realizability based only on $\overline{u_{\alpha} u_{\alpha}} \rightarrow 0$. Their models do not satisfy joint realizability and, therefore, are not fully realizable when a scalar is present. If we impose these conditions on their models, their coefficients must be changed such that their models will reduce to the same form as proposed here. Reynolds (1987) obtained a set of model constraints using 2D-2C conditions on $X_{p j q i}$ which also ensure realizability. His model keeps fourth powers of $b_{i j}$ and, therefore, is more general. However, regardless of the complexity of the model form, the comparison between his model and the DNS data did not show significant improvement over the other models. 


\section{Comparison between Models and Experiments}

For model evaluation we will use the homogeneous shear flow experiment of Tavoularis and Corrsin (1981), DNS of the same flow by Rogers et al (1986) and the buoyant plume experiment of Shabbir and George (1990) and Shabbir (1991). Note that for the homogeneous shear flow the buoyancy effects are negligible. For the experiments the pressure terms were obtained as the closing terms in the balances for the Reynolds stress and heat flux equations. The pressure correlations obtained in this fashion will certainly have some error but this is the closest we can come in obtaining these from the experiment. Also a direct comparison between these and their models is still a better approach in establishing their accuracy rather than solving the differential equations and then comparing the mean flow results etc. with the experiments.

\subsection{Pressure Strain Correlation}

First we look at the comparison between the pressure strain models and the experiment of Tavoularis and Corrsin (1981). The velocity field of this experiment is essentially the same as that of Harris et (1977) and, therefore, the results presented here are applicable to that experiment as well. Figure (1) shows a comparison between the experiment and five different models. The two complete linear models are Launder et al (1975) (LRR) and Zeman and Lumley (1976) and these are of the exact same form except the model coefficients. Of the two, the Zeman and Lumley model seems to give a better overall agreement with the data. The Shih and Lumley (1985) model uses linear form for the slow term with $\beta$ given by Lumley (1978) and is off by a large margin. This is because $\beta$ of Lumley (1978) was calibrated against flows with negative III whereas for the expcriment under consideration this invariant is positive. When instead the Choi and Lumley (1984) model is used for the slow term, all the pressure strain components are predicted within experimental accuracy. Craft et al (1989) also does a good job in predicting all the components. This is to be expected since the coefficients in their model are determined from the homogeneous shear flow experiment.

Figure (2) compares the slow term models with the DNS (19S6) results of the homogeneous shear flow. Lumley's (1978) linear and the Choi and Limley (1984) nonlinear models give the best agreement with the data. Recall that these two models gave very different results for the experiment of Tavoularis and Corrsin (1981). Unlike that experiment, the Reynolds number in the DNS is much smaller. Both of these models incorporate the similar low Reynolds number behavior and, therefore, give similar overall results. Both the Zeman and Lumley (1976) and LRR (1975) model are off the data. The non-linear model of Craft et al (1989) performs well only for the $\Phi_{x x}$ and $\Phi_{x y}$ components. Figure (3) compares the three different models for the rapid pressure term witl the DNS of Rogers et al (1986). Of the three Shih and Lumley (1985) non-linear model gives the best agreement. The LRR (1975) model performs reasonably only for the $\Pi_{z z}^{r a p i d}$ and $\Pi_{r z}^{r a p i d}$ components. 
We also note that the Craft et. al. model performs better than the LRR model.

Figure (4) compares the total pressure-strain models for the buoyant plume experiment of Shabbir and George (1990). For the streamwise component the Zeman and Lumley (1976) model gives the best agreement. The Shih and Lumley (1985) and LRR (1975) models are off the experiment near the centerline of the plume but arc within experimental uncertainty for the rest of the flow. Craft et. al. (1989) overpredicts while Shih-ChoiLumley underpredicts the experiment. For the radial component Zeman and Lumley (1976) model performs the best and, except for Shih-Choi-Lumley, all the other models give reasonable agreement with the experiment. For the $\Pi_{r z}$ component, which is the most important one, Shih-Choi-Lumley, LRR (1975) and Craft et. al. (1989) are in excellent agreement with the experiment. Zeman and Lumley (1976) is also within experimental uncertainty where as Shih and Lumley (1985) overestimates the experiment. We note that no single model gives good agreement with all the flows. It is our fecling that the source of this is either the coefficients for the slow term or the rapid term ( $a_{2}$, given by equation (3.20) was assigned its limiting value). Further work is uderway in order to sort this out.

\subsection{Pressure Temperature-Gradient Correlation}

Now we compare the models for the pressure-temperature gradient correlations with the same three flows. First we look at the experiment of Tavoularis and Corrsin (1981) (figure 5). For the streamwise correlation Shih and Lumley (1985) and the present model provide the best agreement with the data. The Craft et. al. (1989) model overpredicts the data whereas Zeman and Lumley (1976) underpredicts it. The linear model of Launder (1975) does a reasonable job. For the correlation in the cross-stream direction the present model gives the best result and all the other models underpredict the experiment. Since this experiment was used in adjusting the constants in the present. model, this comparison does not provide a true test for this model.

Figure (6) shows a similar comparison with the DNS of Rogers et. al. (1986) for the same flow. For the streamwise correlation, again Shih and Lumlcy and the present model give the best agreement with the data. The non-linear model of Craft et. al. (1989) and the linear model of Launder (1975) also give reasonable results. For the correlation in the cross-stream direction, however, none of the models predict the data, although at the initial stages of the flow the Launder model does a satisfactory job. However,toward the last part of the simulation, where the simulation is more developed, the Launder (1975) model underestimates the simulation by $40 \%$. At the same location the present model overestimates the data by the same amount.

The same models are compared with the buoyant plume expcriment of Shabbir and George (1990) in figure (7). For the streamwise component we note that the present model gives excellent agreement with the experiment. Both Shil and Lumley (1985) and Zeman and Lumley (1976) models also do a reasonable job whercas the rest of the models 
underpredict the experiment. For the radial component, $\Pi_{r}$, the present model somewhat underpredicts the data near the central part of the plume, but produces the correct peak value and gives a very satisfactory overall agreement with the experiment. The rest of the models are off by a large margin. Note that the prediction of the radial component is extremely important for the correct calculation of the mean buoyancy field.

Acknowledgements: AS would like to acknowledge the support of the National Research Council and the Lewis Research Academy of NASA Lewis Rescarch Center for carrying out some of the work reported here. The contribution of JLL was supported in part by the U.S. Air Force Office of Scientific Research under Contract No. AFOSR 89-0226, in part by the U.S. National Aeronautics and Space Administration, Langley Research Center, under Contract No., NAG-1-954, and in part by the U.S. National Science Foundation under Grants Nos. DMS-88-14553 and MSM 86-11164. 


\section{References}

Choi, K.-S. 1983. A Study of the Return to Isotropy of Homogeneous Turbulence. Ph.D. Dissertation, Cornell University.

Choi, K.-S. and J.L. Lumely 1984.. Return to Isotropy of Homogeneous Turbulence Revisited, Turbulence and Chaotic Phenomena in Fluids, ed. T. Tatsumi, Elsevier, North Holland.

Craft T.J. S. Fu, B.E. Launder and D.P. Tselepidakis 1989. Developments in Modelling the Turbulent Second-Moment Pressure Correlations. Report TFD/89/1, Mech. Eng. Dept., UMIST.

Gence, J.N. and J. Mathieu 1980. The Return to Isotropy of a Homogeneous Turbulence Having Been Submitted to Two Successive Plane Strains. J. Fluicl Mech., Vol 101, 556-566.

Harris, V.G., J.A.H. Graham and S. Corrsin 1977. Further Expcriments in Nearly Homogeneous Shear Flow. J. Fluid Mech., Vol. 81, 657-687.

Launder, B.E. 1975. On the Effects of Gravitational Field on the Turbulent Transport of Heat and Momentum. J. Fluid Mech., Vol 67,569-590.

Launder, B.E. 1989. Phenomenological Modeling: Present and Future? Whither Turbulence? Cornell University, March 22-24.

Launder, B.E., Reece, G.L. and Rodi, W. 1975. Progress in the development of a Reynoldsstress turbulence closure. J. Fluid Mech. 68, pp. 537-566.

Lumley, J.L. 1978. Computational modeling of turbulent flows. in Advances in Applied Mechanics, Vol. 18, Academic Press, New York, pp.123-176.

Lumley, J.L. 1983. Turbulence Modeling. ASME Journal of Applied Mechanics, Journal of Applied Mechanics, Vol. 50, pp 1097-1103.

Pope, S.B. 1983. Consistent Modeling of Scalars in Turbulent Flows, Phys. Fluids, Vol. $26,404-408$.

Reynolds, W.C. (1987) Fundamentals of Turbulence for Turbulence Modeling and Simulation. Lecture Notes for Von Karman, March 16-17, 1987.

Reynolds, W.C. (1976) Computation of Turbulent Flows. Ann. Rev. Fluid Mech., Vol 8, 183-208. 
Reynolds, W.C. 1989. Effect of Rotation on Homogeneous Turbulence. Tenth Australian Fluid Mechanics Conference, December 11-15.

Reynolds, W.C. and Grau 1988, personal communication.

Rodi, W. 1988. Recent Developments in Turbulence Modeling. Proc. 3rd Int. Symp. on Refined Flow Modeling and Turbulence Measurements, Tokyo, July 26-28.

Rogers, M.M., P. Moin and W.C. Reynolds 1986. The Structure and Modeling of the Hydrodynamic and Passive Scalar Fields in Homogeneous Turbulent Shear Flow. Report No. TF-25, Department of Mech. Engg., Stanford Univ.

Schumann, U. 1977. Realizability of Reynolds Stress Turbulence Models. Phys. Fluids, $20,721-725$.

Shabbir, A., and W.K. George. 1990. Experimental Study of an Axisymmetric Turbulent Buoyant Plume. Submitted to J. Fluid Mech.

Shabbir, A. 1991. Experimental Balances of the Second Moment Equations for the Axisymmetric Buoyant Plume and Its Implications on Turbulence Modeling. Submitted to the 8th Symposium on Turbulent Shear Flows to be held in 1991.

Shih, T.H. and J.L. Lumley. 1986 Influence of Timescale Ratio on Scalar Flux Relaxation: Modeling Sirivat and Warhaft's Homogeneous Passive Scalar Fluctuations, J. Fluid Mech., Vol. 162, 211-222.

Shih, T.H., J.L. Lumley and J.Y. Chen. 1990 Second Order Morcling of a Passive Scalar in a Turbulent Shear Flow, AIAA Journal, Vol. 28, No. 4, 610-617.

Shih, T.H., W.C. Reynolds and N.N. Mansour. 1990. A Spectrum model for Weakly Anisotropic Turbulence. Phys. Fluids A2(8).

Shih, T.h., J.L. Lumley and J.Y. Chen. 1985. Second Order Modeling of Boundary-Free Turbulent Shear Flows with a New Model Form of Pressure Correlations. FDA-85-07, Sibley School of Mech. and Aero. Engg., Cornell University.

Shih, T.H., N.N. Mansour and J.-Y. Chen. 1987. Reynolds Stress Models of Homogeneous Turbulence, Proceedings of the Summer Program 1987, Center for Turbulence Research, Stanford University and NASA Ames.

Shih, T.H. and Lumley, J.L. 1985. Modeling of pressure correlation terms in Reynoldsstress and scalar flux equations. Rept. FDA-85-3, Sibley School of Mech. and Aerospace Eng., Cornell University. 
Speziale, C.G. 1983.Closure Models for Rotating Two-dimensional Turbulence. Geophys. and Astrophys. Fluid Dynamics, 23,69.

Speziale, C.G., N.N. Mansour and R.S. Rogallo. 1987. The Decay of Isotropic Turbulence in a Rapid Rotating frame. CTR-S87-12. Stanford University and NASA Ames Research Center.

Tavoularis, S. and S. Corrsin. 1981. J. Fluid Mech., Experiments in Nearly Homogeneous Turbulent Shear Flow with a Uniform Mean Temperature Gradient. Part 2. The Fine Structure. Vol 104, 349-367.

Tennekes, H. and J.L. Lumley. 1972. A First Course in Turbulence, MIT Press, Cambridege, Massachusetts.

Zeman, O. 1981. Progress in the Modeling of Planetary Boundary Layers. Ann. Rev. Fluid Mech., Vol. 13, 253-272.

Zeman, O. and J.L. Lumley. 1976. Modeling Buoyancy Driven Mixed Layers. J. Atm. Sci., Vol. 33. 


\section{APPENDIX A}

The following are the exact expressions for the rapid and buoyancy contributions to the pressure-strain and pressure scalar-gradient correlations.

$$
\begin{aligned}
X_{p j q i} & =-(1 / 4 \pi) \iiint_{v}\left[\overline{u_{q}(r) u_{i}\left(r^{\prime}\right)}\right]_{, p j} d v /\left|r-r^{\prime}\right| \\
X_{i j k} & =-(1 / 4 \pi) \iiint_{v}\left[\overline{u_{k}(r) \theta_{j}\left(r^{\prime}\right)}\right]_{, j i} d v /\left|r-r^{\prime}\right| \\
Y_{k i j} & =-(1 / 4 \pi) \iiint_{v}\left[\overline{\left.u_{j}(r) \theta\left(r^{\prime}\right)\right]_{, k i} d v /\left|r-r^{\prime}\right|}\right. \\
Y_{i k} & =-(1 / 4 \pi) \iiint_{v}\left[\overline{\theta(r) \theta\left(r^{\prime}\right)}\right]_{, i k} d v /\left|r-r^{\prime}\right|
\end{aligned}
$$

The slow terms are defined by the following expressions

$$
\begin{aligned}
-\Phi_{i j} \epsilon & =(1 / \rho) \overline{p_{s l}\left(u_{i, j}+u_{j, i}\right)}-2 \nu \overline{u_{i, k} u_{j, k}}+2 \epsilon \delta_{i j} / 3 \\
-\Phi_{i} \epsilon / \overline{q^{2}} & =(1 / \rho) \overline{p_{s l} \theta_{, i}}-(\nu+\gamma) \overline{\theta_{, k} u_{i, k}}
\end{aligned}
$$

The total (i.e. sum of the slow, rapid and buoyancy parts) pressure strain and pressure scalar-gradient correlation has been denoted by $\Pi_{i j}$ and $\Pi_{i}$ respectively. 


\section{APPENDIX B}

The values of the model coefficients $\beta$ 's and $\gamma$ 's as calculated from the buoyant plume experiment of Shabbir and George (1990)

$\begin{array}{ccccccccccc}r / z & \beta_{1} & \beta_{2} & \beta_{3} & \beta_{4} & \beta_{5} & \beta_{6} & \beta_{7} & \beta_{8} & \beta_{9} & \beta_{11} \\ 0.00 & 0.47 & -0.12 & -2.05 & -0.35 & 0.65 & -0.20 & 6.40 & 1.95 & -7.05 & -1.30 \\ 0.01 & 0.47 & -0.12 & -2.04 & -0.35 & 0.65 & -0.20 & 6.20 & 1.94 & -6.85 & -1.30 \\ 0.02 & 0.48 & -0.12 & -2.02 & -0.36 & 0.64 & -0.19 & 5.72 & 1.93 & -6.36 & -1.28 \\ 0.03 & 0.49 & -0.12 & -1.99 & -0.37 & 0.63 & -0.18 & 5.16 & 1.90 & -5.79 & -1.27 \\ 0.04 & 0.50 & -0.12 & -1.96 & -0.37 & 0.63 & -0.17 & 4.66 & 1.88 & -5.29 & -1.25 \\ 0.05 & 0.51 & -0.13 & -1.93 & -0.38 & 0.62 & -0.16 & 4.28 & 1.85 & -4.90 & -1.24 \\ 0.06 & 0.52 & -0.13 & -1.91 & -0.39 & 0.61 & -0.15 & 4.01 & 1.83 & -4.62 & -1.22 \\ 0.07 & 0.53 & -0.13 & -1.88 & -0.40 & 0.60 & -0.14 & 3.83 & 1.81 & -4.44 & -1.21 \\ 0.08 & 0.53 & -0.13 & -1.86 & -0.40 & 0.60 & -0.13 & 3.73 & 1.80 & -4.33 & -1.20 \\ 0.09 & 0.54 & -0.14 & -1.84 & -0.41 & 0.59 & -0.13 & 3.69 & 1.78 & -4.28 & -1.19 \\ 0.10 & 0.55 & -0.14 & -1.83 & -0.41 & 0.59 & -0.12 & 3.68 & 1.77 & -4.27 & -1.18 \\ 0.11 & 0.55 & -0.14 & -1.81 & -0.41 & 0.59 & -0.11 & 3.70 & 1.76 & -4.28 & -1.17 \\ 0.12 & 0.56 & -0.14 & -1.80 & -0.42 & 0.58 & -0.11 & 3.74 & 1.75 & -4.32 & -1.16 \\ 0.13 & 0.56 & -0.14 & -1.79 & -0.42 & 0.58 & -0.11 & 3.78 & 1.74 & -4.36 & -1.16 \\ 0.14 & 0.56 & -0.14 & -1.79 & -0.42 & 0.58 & -0.11 & 3.83 & 1.74 & -4.41 & -1.16 \\ 0.15 & 0.56 & -0.14 & -1.80 & -0.42 & 0.58 & -0.11 & 3.87 & 1.74 & -4.45 & -1.16 \\ 0.16 & 0.56 & -0.14 & -1.81 & -0.42 & 0.58 & -0.11 & 3.91 & 1.75 & -4.49 & -1.17 \\ 0.17 & 0.55 & -0.14 & -1.83 & -0.41 & 0.59 & -0.12 & 3.93 & 1.77 & -4.51 & -1.18 \\ 0.18 & 0.54 & -0.13 & -1.85 & -0.40 & 0.60 & -0.13 & 3.93 & 1.79 & -4.53 & -1.19\end{array}$

$\begin{array}{cccccc}r / z & \gamma_{1} & \gamma_{2} & \gamma_{3} & \gamma_{4} & \gamma_{5} \\ 0.00 & 0.41 & -2.82 & -0.36 & -0.81 & -3.15 \\ 0.01 & 0.41 & -2.75 & -0.35 & -0.81 & -2.96 \\ 0.02 & 0.42 & -2.58 & -0.34 & -0.80 & -2.51 \\ 0.03 & 0.42 & -2.39 & -0.32 & -0.79 & -1.99 \\ 0.04 & 0.43 & -2.22 & -0.30 & -0.79 & -1.53 \\ 0.05 & 0.43 & -2.10 & -0.28 & -0.79 & -1.19 \\ 0.06 & 0.44 & -2.01 & -0.27 & -0.81 & -0.95 \\ 0.07 & 0.44 & -1.95 & -0.27 & -0.83 & -0.81 \\ 0.08 & 0.44 & -1.92 & -0.28 & -0.87 & -0.74 \\ 0.09 & 0.44 & -1.91 & -0.30 & -0.93 & -0.72 \\ 0.10 & 0.44 & -1.91 & -0.33 & -1.00 & -0.73 \\ 0.11 & 0.44 & -1.92 & -0.37 & -1.09 & -0.77 \\ 0.12 & 0.45 & -1.94 & -0.41 & -1.21 & -0.83 \\ 0.13 & 0.45 & -1.96 & -0.47 & -1.35 & -0.88 \\ 0.14 & 0.44 & -1.98 & -0.53 & -1.53 & -0.93 \\ 0.15 & 0.44 & -2.00 & -0.59 & -1.75 & -0.97 \\ 0.16 & 0.44 & -2.02 & -0.64 & -2.02 & -0.99 \\ 0.17 & 0.43 & -2.03 & -0.69 & -2.35 & -0.98 \\ 0.18 & 0.42 & -2.05 & -0.71 & -2.76 & -0.95\end{array}$




\section{APPENDIX C}

Here we list the various model coefficients for the slow terms used in the present study. The coefficient $\beta$ used in the Shih and Lumley (1985) model is the one suggested by Lumley (1978) and is

$$
\begin{aligned}
\beta= & 2+\exp \left[-7.77 / R_{l}^{1 / 2}\right] \\
& \left\{72 / R_{l}^{1 / 2}+80.1 \ln [1+62.4(-I I+2.3 I I I)]\right\}\left(\frac{1}{9}+3 I I I+I I\right)
\end{aligned}
$$

The function $H$ appearing in (3.29) and (3.30) is taken from Shih et. al. (1990) and is given by

$$
H=1.1+0.55(\beta-1) \tanh \left[4\left(\frac{\tau}{\tau_{\theta}}-1\right)\right]
$$

The coefficients $\beta$ and $\gamma$ appearing in equation (3.27) and suggested by Choi and Lumley (1984) are given by

$$
\begin{aligned}
& \beta=2+\frac{\rho^{*} F^{1 / 2}}{\left(1+G \chi^{2}\right)} \\
& \gamma=\frac{\rho^{*} F^{1 / 2}}{\left(1+G \chi^{2}\right)} \frac{G}{\xi}
\end{aligned}
$$

where

$$
\begin{aligned}
\xi & =(I I I / 2)^{1 / 3}, \quad \eta=(-I I / 3)^{1 / 2} \\
\chi & =\frac{\xi}{\eta}, \quad G=-\chi^{4}+0.8 \chi^{6} \\
\rho^{*} & =\exp \left[-9.29 / R_{l}^{1 / 2}\right]\left\{\left(\frac{7.69}{R_{l}^{1 / 2}}+\frac{73.7}{R_{l}}\right)-\left[296-16.2(\lambda+1)^{4}\right] I I\right\}
\end{aligned}
$$

For the Zeman and Lumely (1976) model following set of coefficients were used

$$
\begin{aligned}
\beta & =\frac{1}{(-15 I I)}\left[1+(1-24 I I)^{1 / 2}\right] \\
f_{1} & =\left(\frac{\beta}{2}+\frac{\overline{q^{2}} \epsilon_{\theta}}{\epsilon \bar{\theta}^{2}}\right)
\end{aligned}
$$

Note that these revised coefficients are taken from Zeman (1981) and are different from the values originally used by Zeman and Lumley (1976). It should also be pointed out that the expression for $\beta$ is clipped at 6.0. 

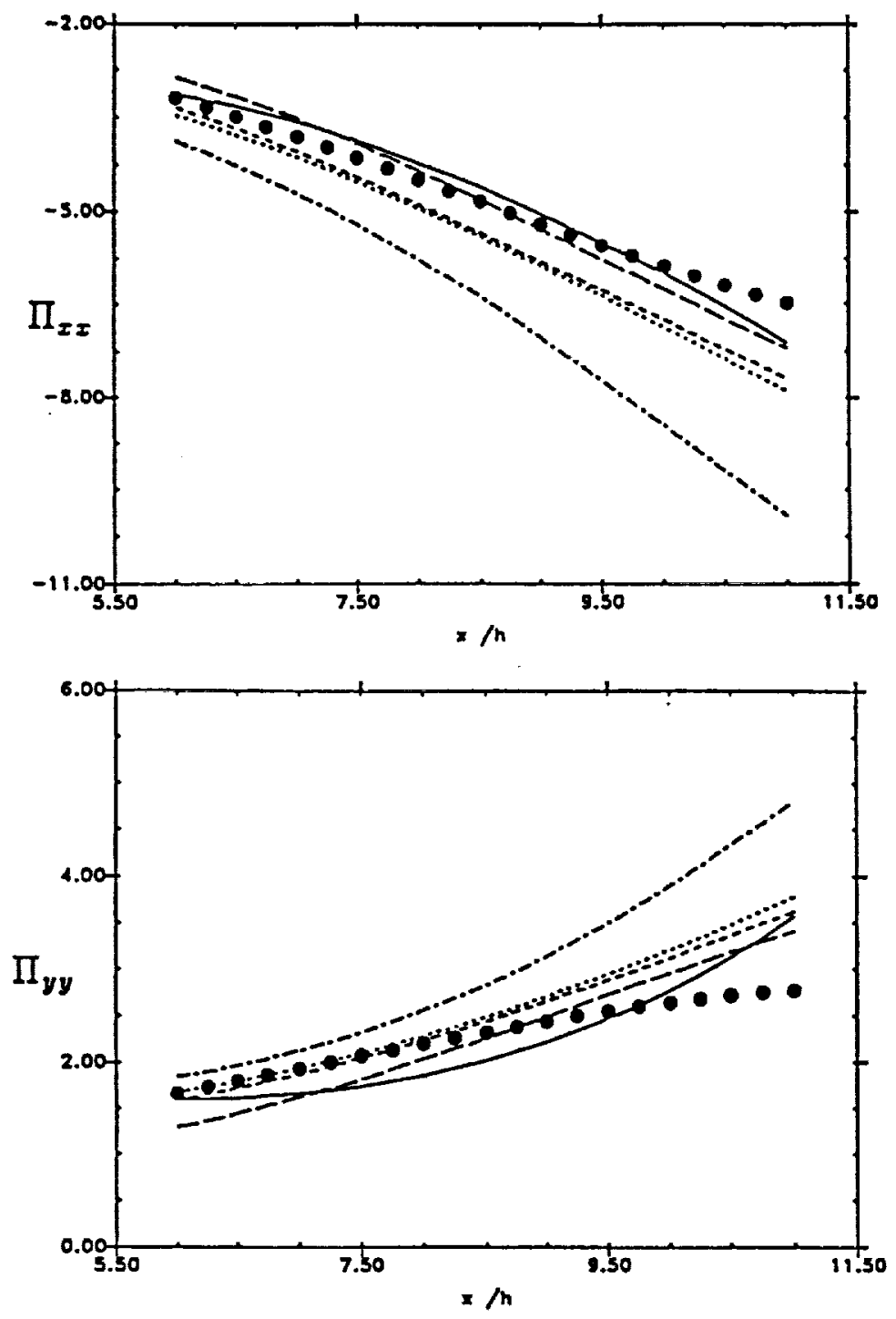

Figure 1. Comparison between models for $\Pi_{i j}$ and the homogeneous shear flow experiment. ...... LRR model; -..- Zeman and Lumley model (1976); - - - Shih and Lumley (1985) model;---Craft et. al. (1989) model; — Shih-Choi-Lumley model; $\bullet$ experiment of Tavoularis and Corrsin (1981). 

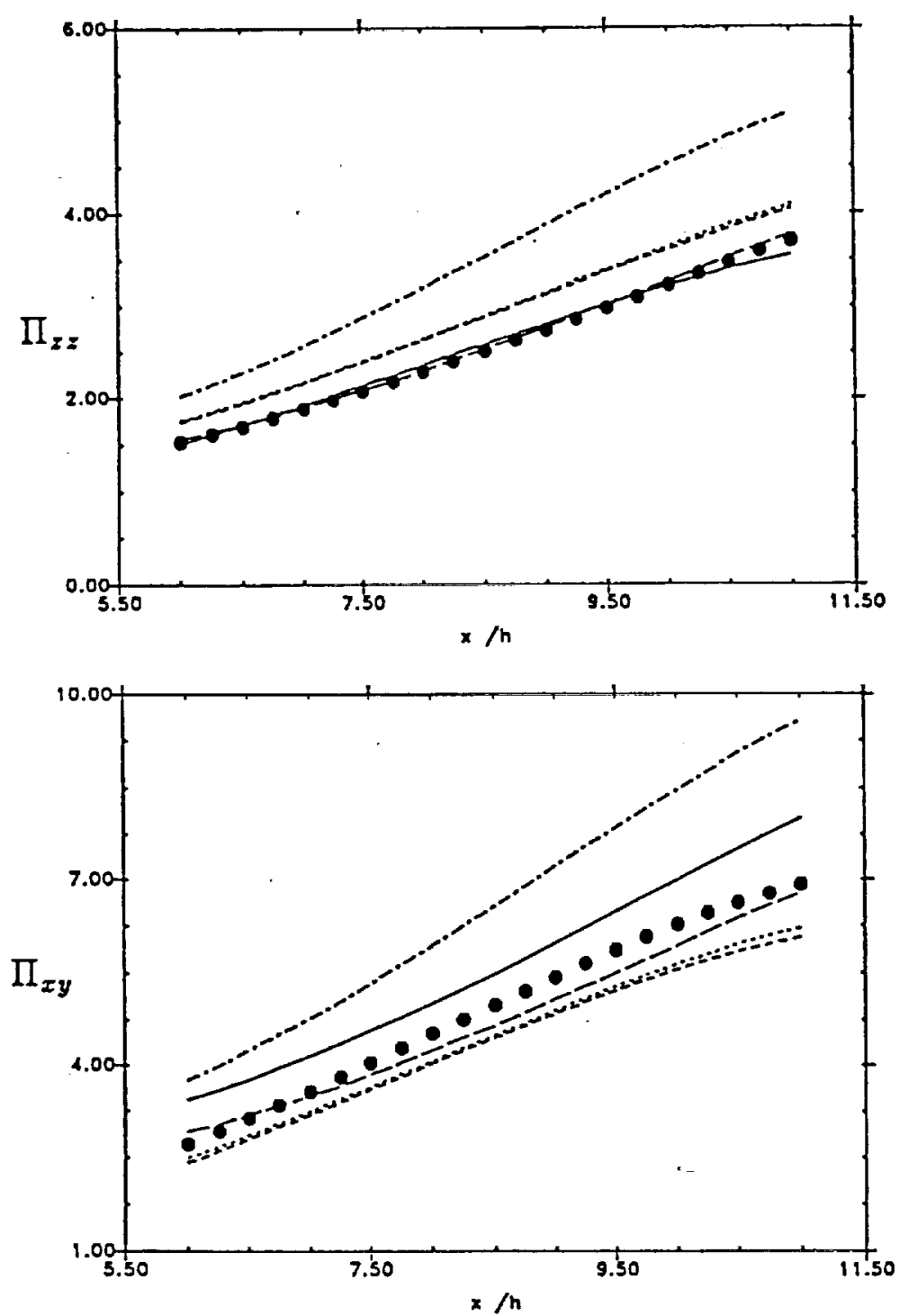

(Figure 1 continued from last page) 

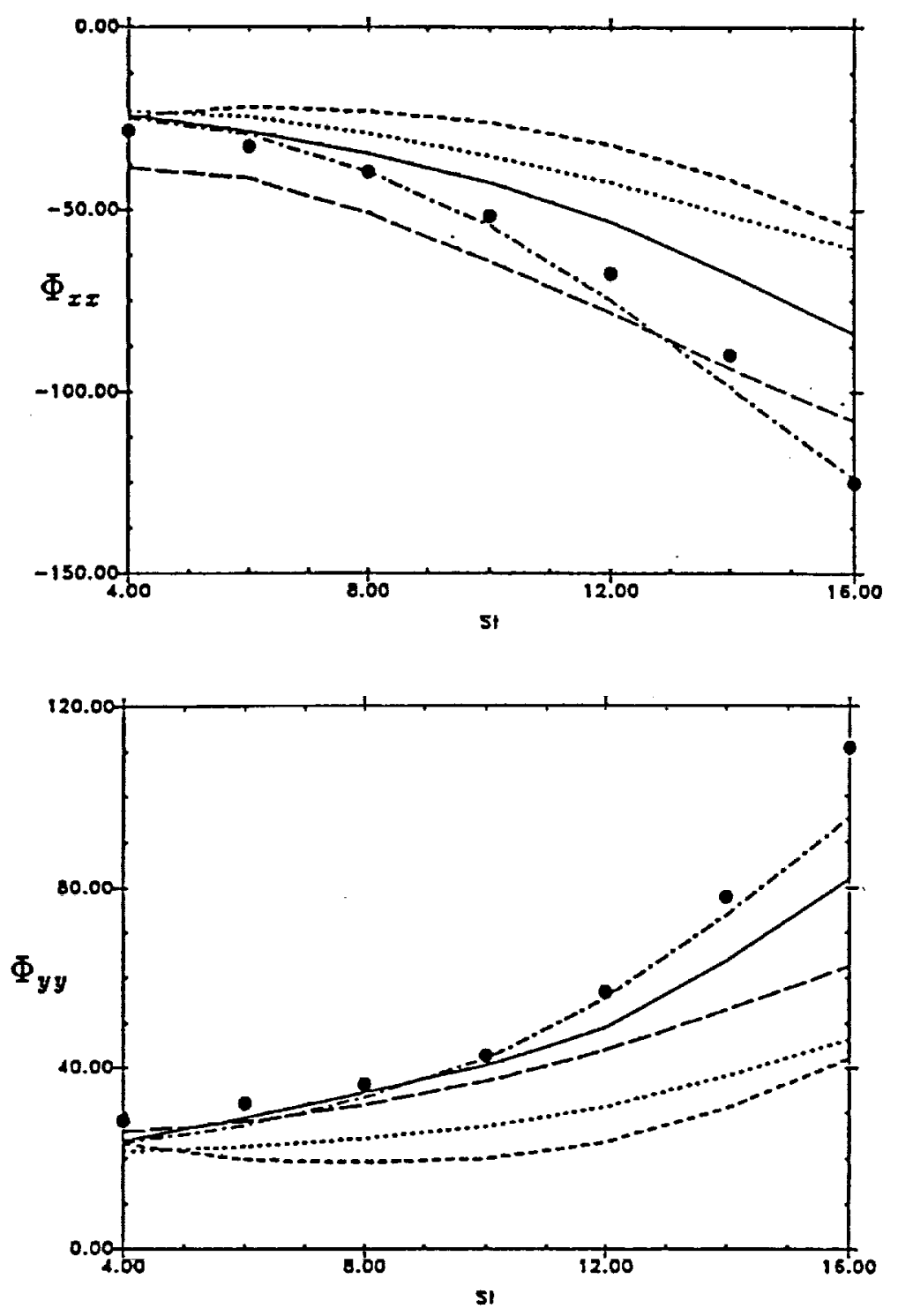

Figure 2. Comparison between the models of the slow part of the pressure-strain and the DNS of homogeneous shear flow. ....... LRR model; -..- Zeman and Lumley model (1976);-..-Shih and Lumley (1985) model;---Craft et. al. (1989) model; - Shih-Choi-Lumley model; $\bullet$ DNS of Rogers et. al. (1986). 

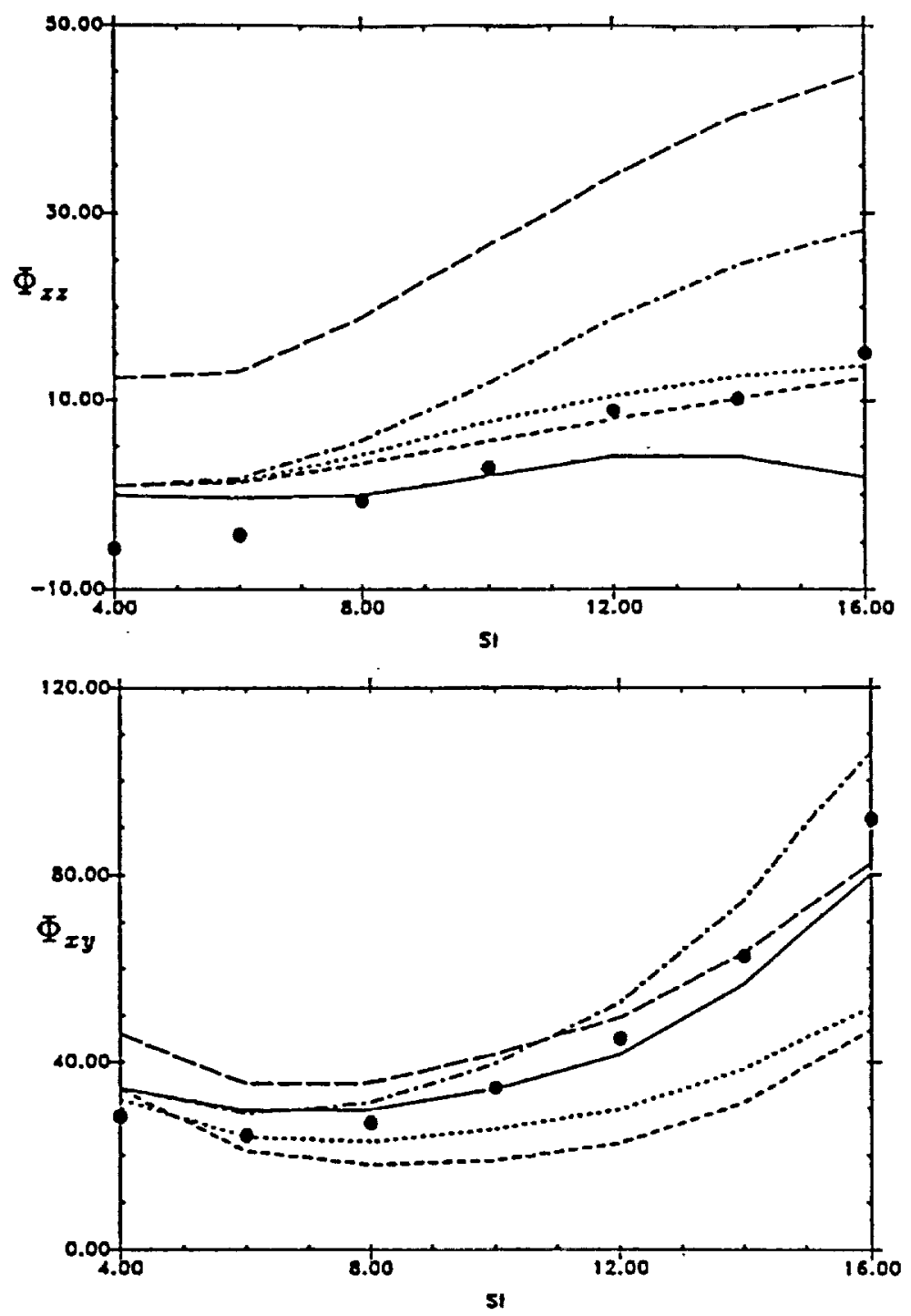

(Figure 2 continued from last page) 

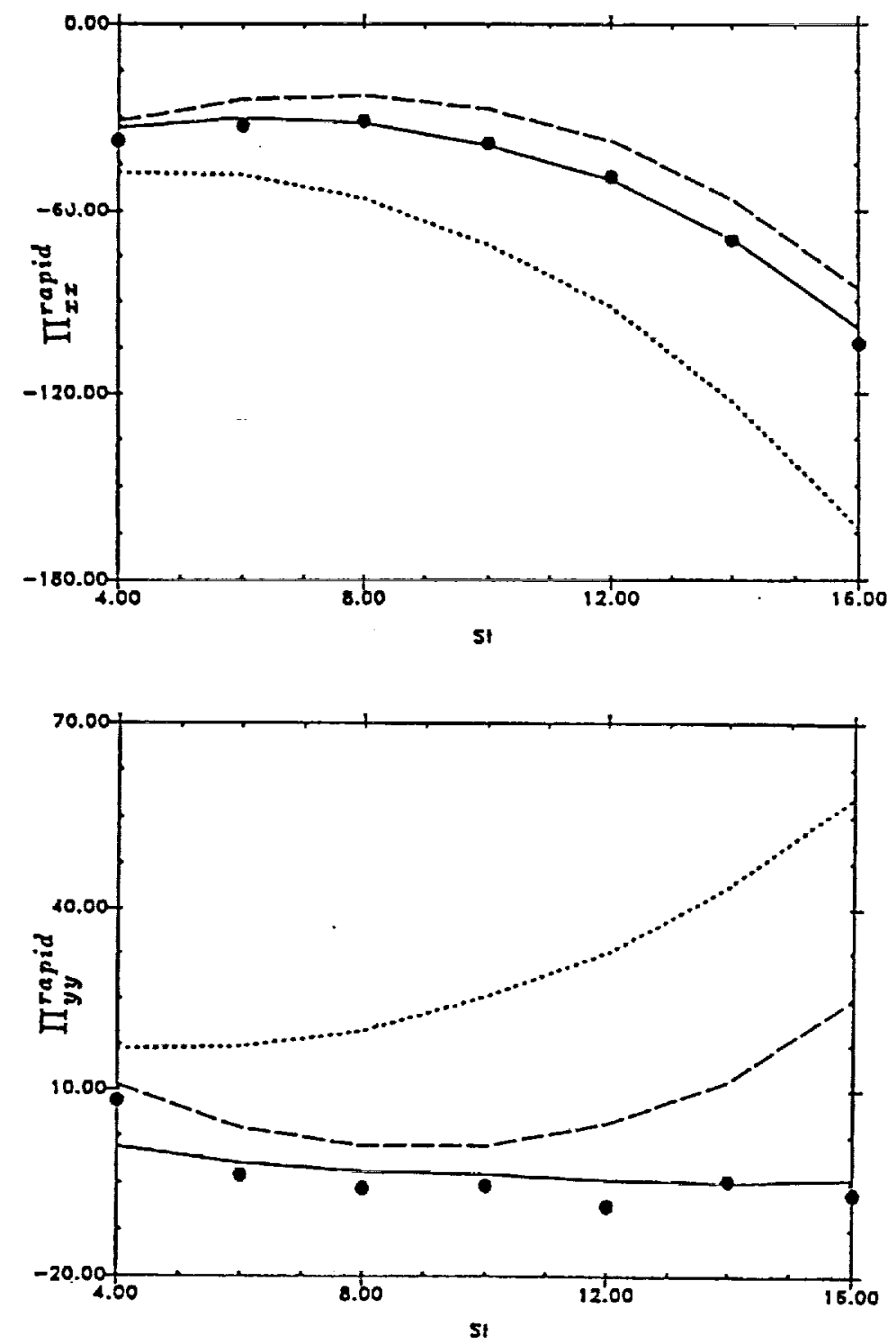

Figure 3. Comparison between the models of the rapid part of the pressure-strain and the DNS of homogeneous shear flow. ..... LRR model; —- Shih and Lumley (1985) model; -- -- Craft et. al. (1989) model; • DNS of Rogers et. al. (1986). 

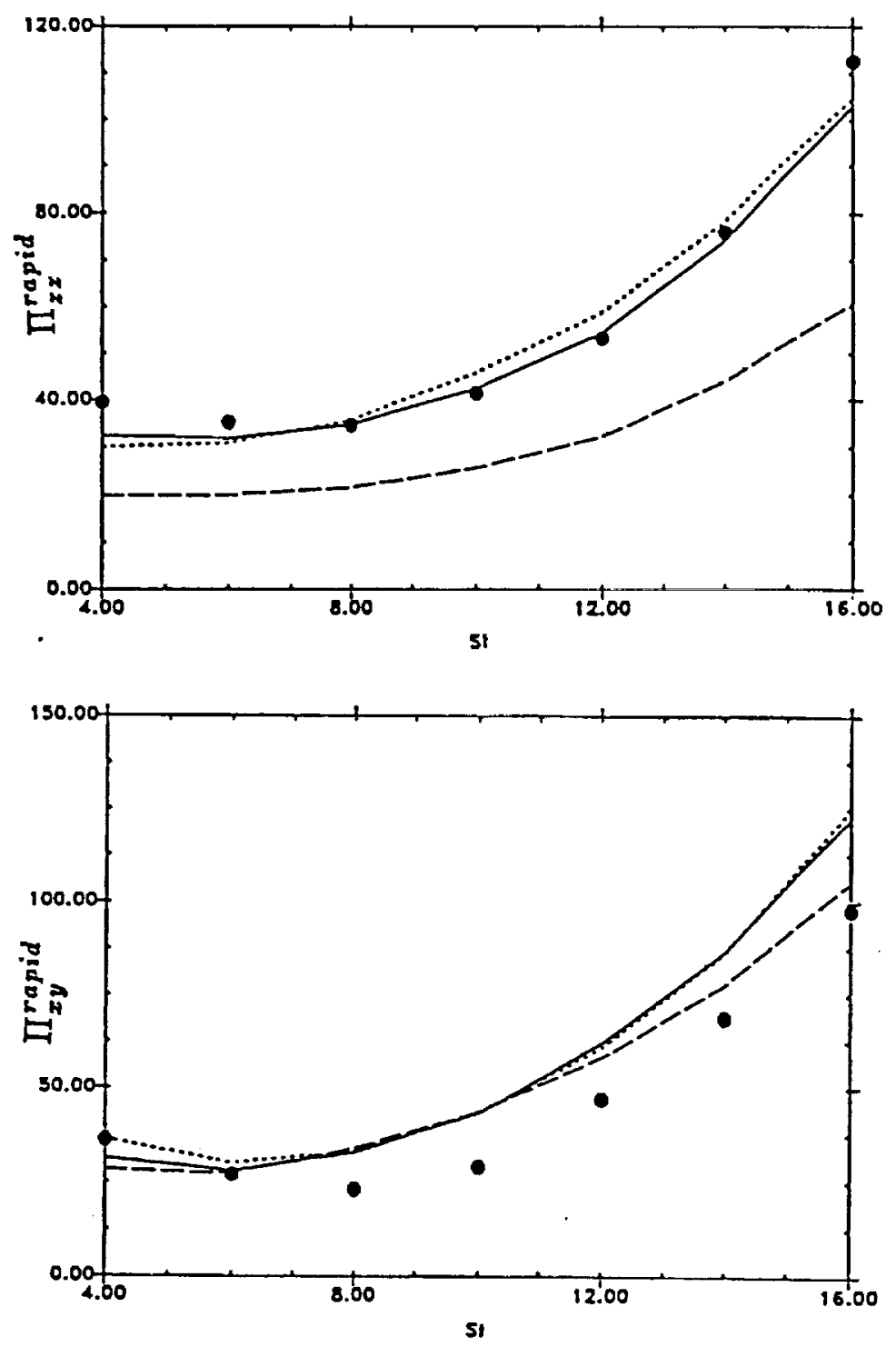

(Figure 3 continued from last page) 

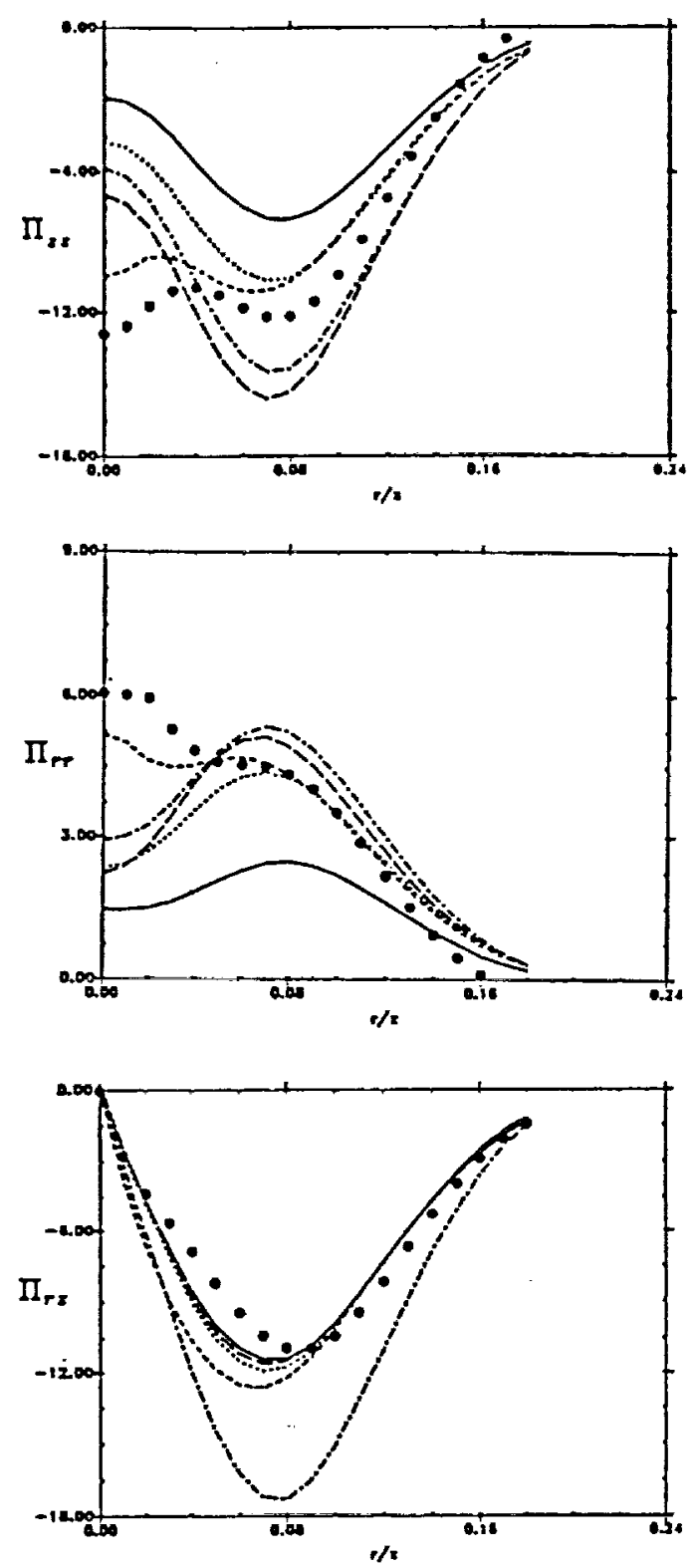

Figure 4. Comparison between models for $\Pi_{i j}$ and the buoyant plume experiment. ....... . LRR model; ...... Zeman and Lumley model (1976); ...... Shih and Lumley (1985) model;--- Craft et. al. (1989) model; — Shih-Choi-Lumley model; • experiment of Shabbir and George (1990). 

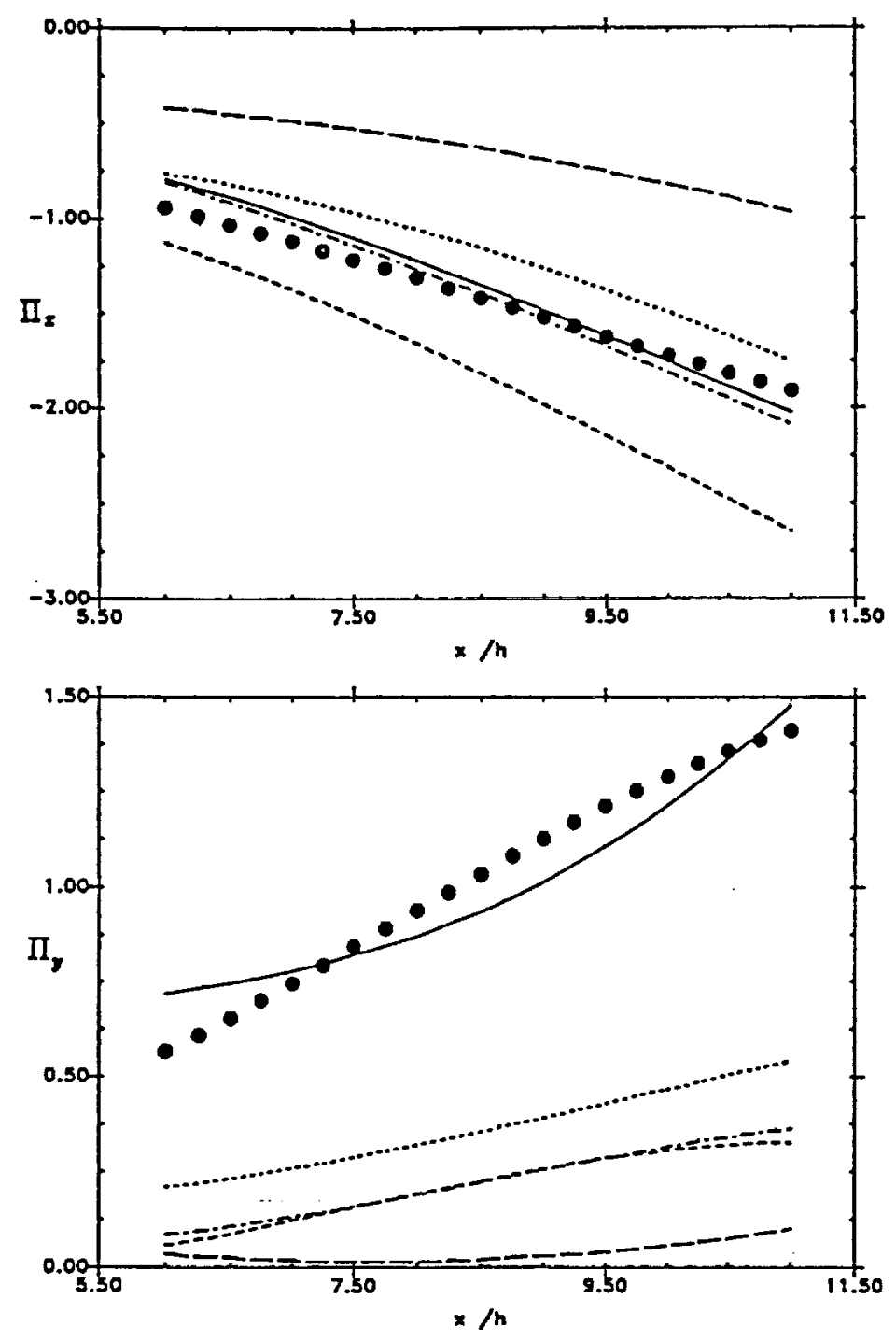

Figure 5. Comparison between models for $\Pi_{i}$ and the homogeneous shear flow experiment. ...... Launder (1975) model;--.-. Zeman and Lumley model (1976);-.-Shih and Lumley (1985) model; - - Craft et. al. (1989) model; —— present model; • experiment of Tavoularis and Corrsin (1985). 

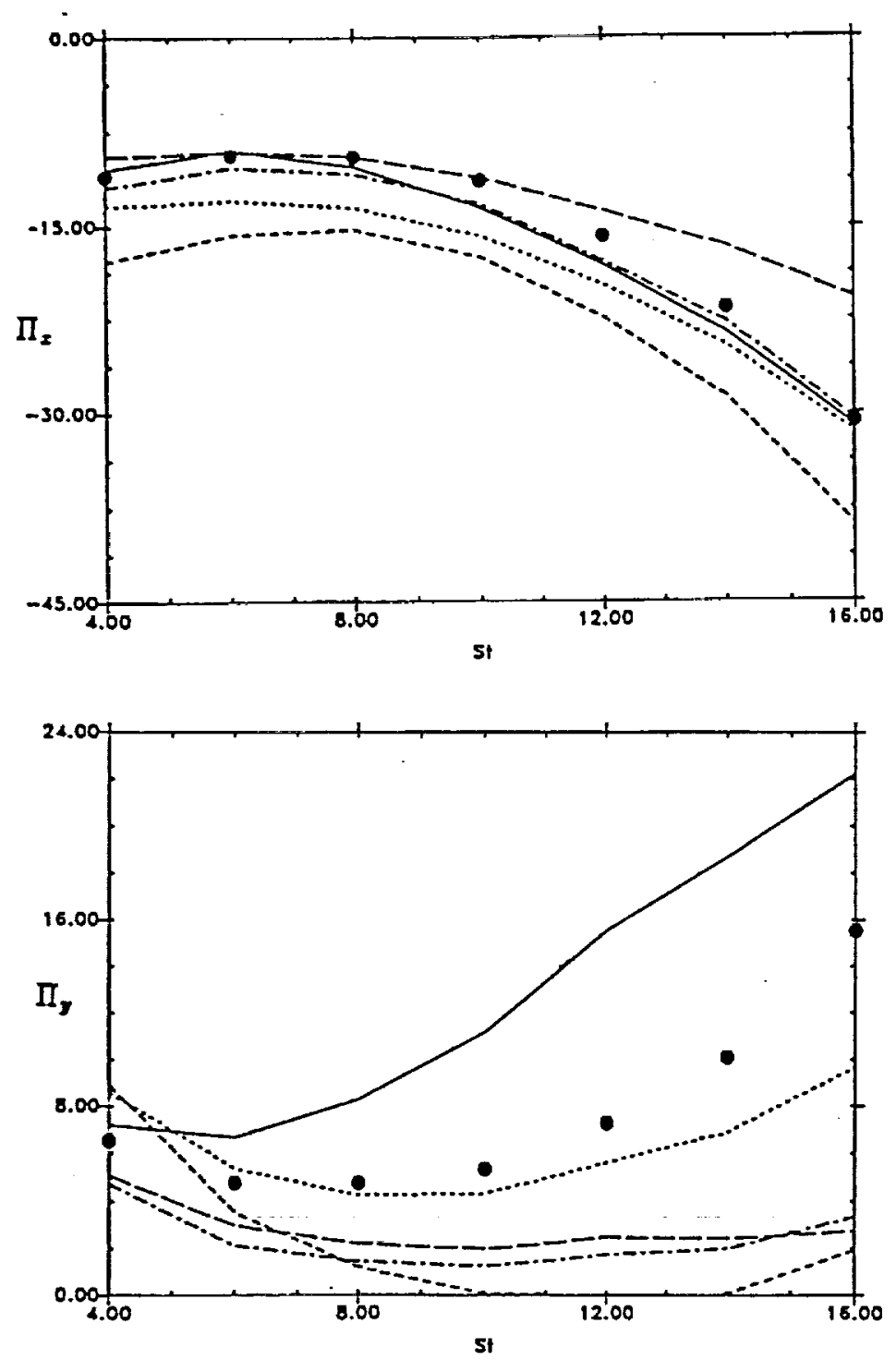

Figure 6. Comparison between models for the $\Pi_{i}$ and the DNS of homogeneous shear flow. .......Launder (1975) model; -.... Zeman and Lumley model (1976); - - - Shih and Lumley (1985) model; -- Craft et. al. (1989) model; —_ present model; • DNS of Rogers et. al. (1986). 

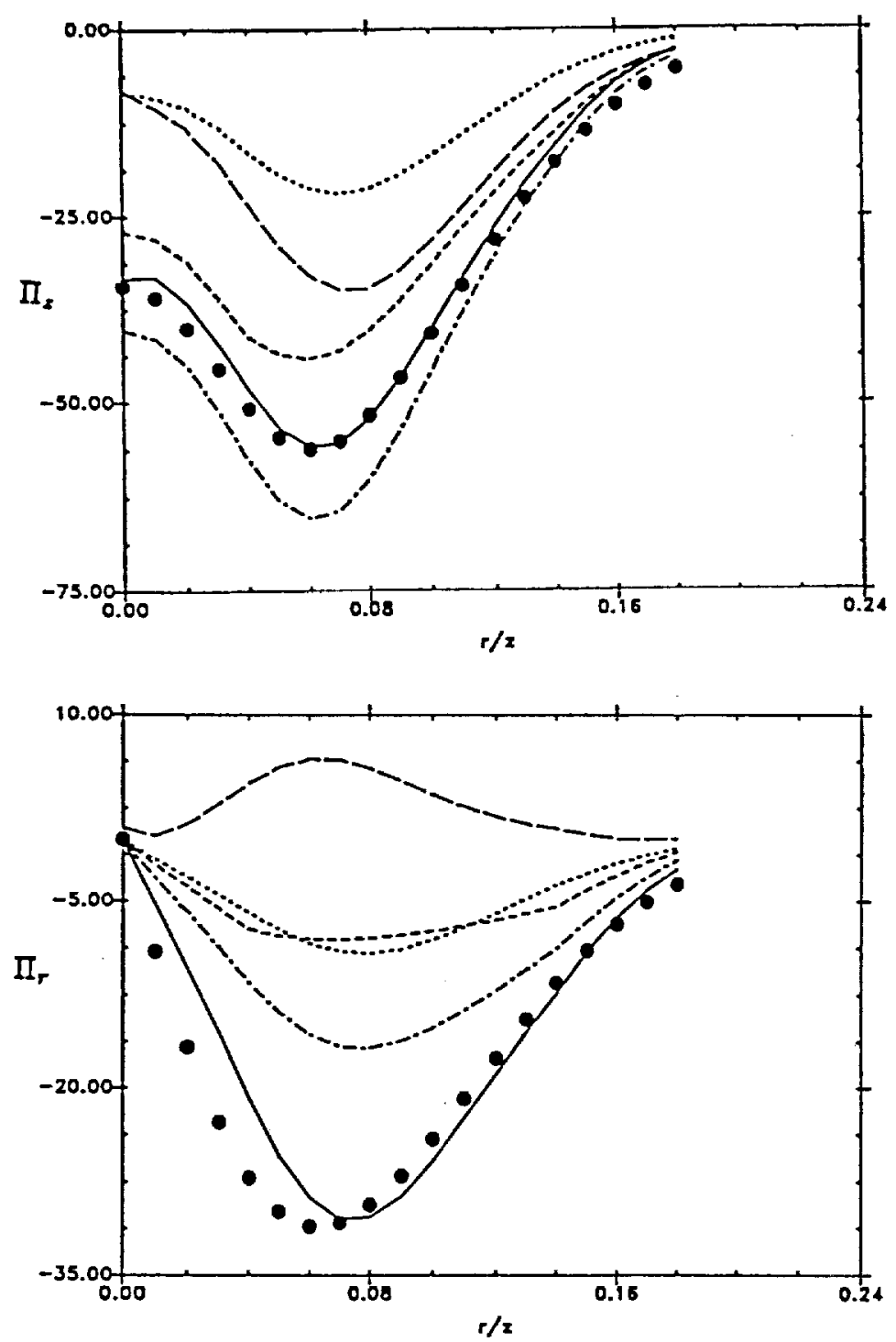

Figure 7. Comparison between models for $\Pi_{i}$ and the buoyant plume experiment. ....... Launder (1975) model; -... .Zeman and Lumley model (1976); - - Shih and Lumley (1985) model; -- Craft et. al. (1989) model; — present model; $\bullet$ experiment of Shabbir and George (1990). 


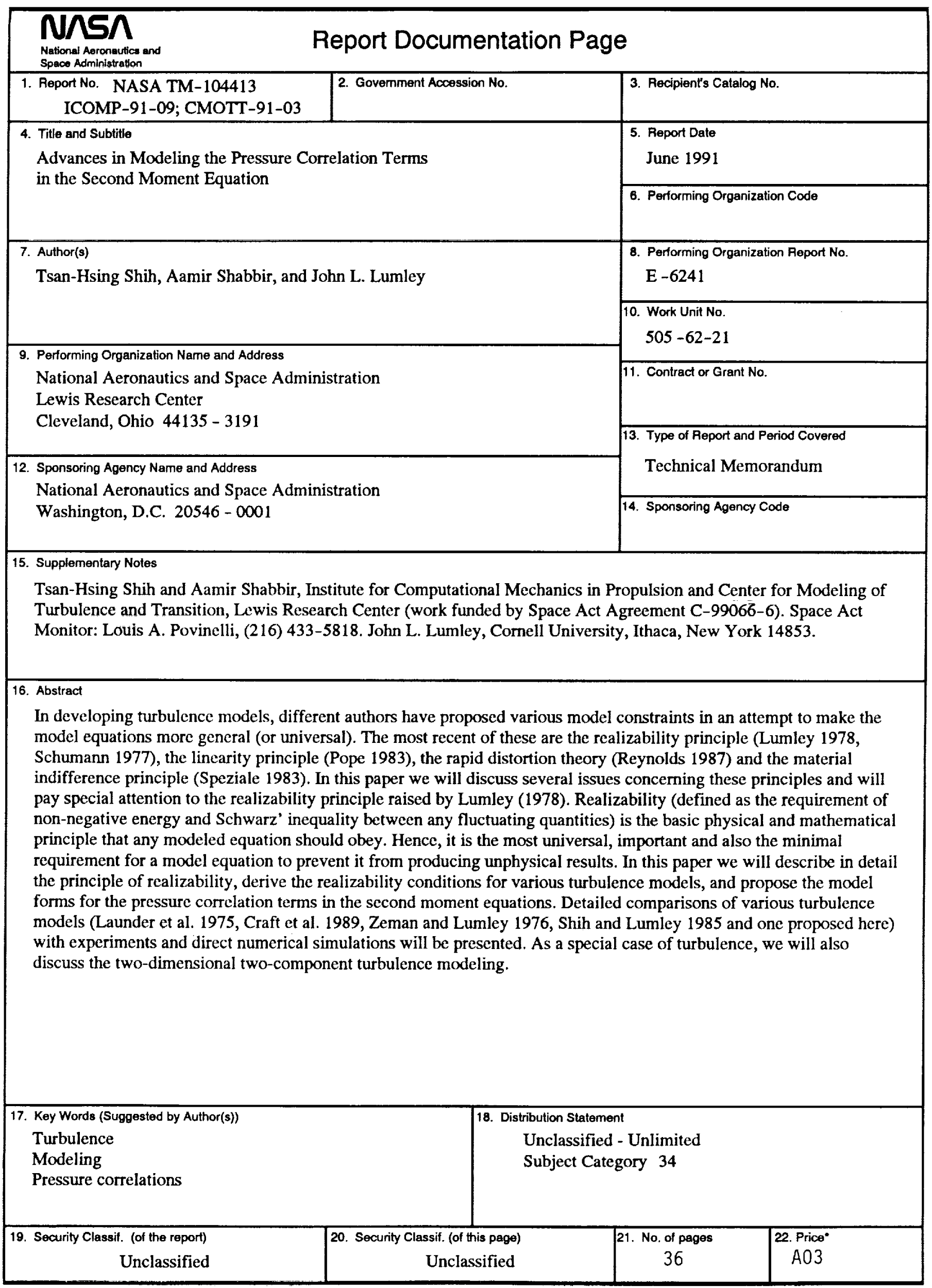

